University of Zurich

Department of Economics

Working Paper Series

ISSN 1664-7041 (print)

ISSN 1664-705X (online)

Working Paper No. 318

\title{
Multi-Battle Contests, Finite Automata, and the Tug-of-War
}

Christian Ewerhart and Julian Teichgräber

February 2019 


\title{
Multi-Battle Contests, Finite Automata, and the Tug-of-War
}

\author{
Christian Ewerhart* Julian Teichgräber**
}

February 25, 2019

\begin{abstract}
This paper examines multi-battle contests whose extensive form can be represented in terms of a finite state machine. We start by showing that any contest that satisfies our assumptions decomposes into two phases, a principal phase (in which states cannot be revisited) and a concluding tie-breaking phase (in which all non-terminal states can be revisited). Degenerate cases are the finite-horizon contests on the one hand (e.g., the match race), and the tug-of-war on the other. Next, assuming a probabilistic technology in each battle, we show that any contest satisfying our assumptions, with either finite or infinite horizon, admits a unique symmetric and interior Markov perfect equilibrium. This entails, in particular, a complete characterization of the equilibrium in the tug-of-war. Finally, we explore, both analytically and numerically, the intricate problem of a contest designer that maximizes expected total effort. In the absence of a complexity constraint, the revenue-maximizing contest is always a match race, where the optimal length of the race increases as the technology of the component contest becomes more noisy. If, however, the complexity constraint is binding, then the optimal contest is typically (but not always) a tug-of-war.
\end{abstract}

Keywords. Dynamic contests $\cdot$ Finite automata $\cdot$ Match race $\cdot$ Tug-of-war $\cdot$ Markov perfect equilibrium . Contest design

JEL-Codes. C62 - Existence and Stability Conditions of Equilibrium; C63 - Computational Techniques, Simulation Modeling; C72 - Noncooperative Games; D72 - Political Processes: Rent-Seeking, Lobbying, Elections, Legislatures, and Voting Behavior

*) (corresponding) Department of Economics, University of Zurich, Schönberggasse 1, CH-8001 Zurich, Switzerland; christian.ewerhart@econ.uzh.ch.

**) Zurich GSE, University of Zurich; julian.teichgraeber@econ.uzh.ch. 


\section{Prerequisites}

\subsection{Introduction}

In a large variety of environments, dynamic contests arise as a natural form of interaction between two competing parties (Fudenberg et al., 1983; Harris and Vickers, 1987; Roberts and Samuelson, 1988; Hörner, 2004; Konrad and Kovenock, 2005, 2009; Agastya and McAfee, 2006; Klumpp and Polborn, 2006; Doraszelski and Markovich, 2007; Fu et al., 2015; Häfner, 2017; Karagözoglu et al., 2018). Given the temporal dimension of the interaction, the winner of the bilateral competition is not determined immediately, but through a potentially indefinite sequence of individual battles. While the literature has focused mainly on the important special cases of the match race and the tug-of-war, a dynamic contest may in general be governed by a more elaborate set of rules. For example, while initially, a start-up may be able to use its seed capital for cost-intensive R\&D, it may be forced to give up at a later stage if lagging behind too much. Similar forms of dynamic contests, in which the conditions for winning change over time, can be found, for instance, in prolonged political conflict (e.g., Organski and Lust-Okar, 1997), biological struggles (e.g., Reeve et al., 1998), and warfare (e.g., Edwards, 2000). Still, a flexible way to model general dynamical conflicts has, to our knowledge, been lacking in the literature. Moreover, the way in which contestants' incentives for effort provision depend on the "rules" of the conflict is, overall, not particularly well-understood.

In this paper, we consider a class of dynamic games in which two players repeatedly face each other in the same type of contest. Following the literature, we will refer to the individual encounter as a component contest (or battle), and to the overall interaction as a (multi-battle) contest. It will be assumed that, at each stage of the contest, the history of victories in prior battles determines if the contest ends and is won by one of the players, or if the contest continues. If the contest ends, then the prize is allocated. Extending the before-mentioned literature on dynamic contests, we assume that the contest rules can be defined in terms of a finite state machine (Moore, 1956; Rubinstein, 1986; Salant, 2011). Since the class of such rules is still quite flexible (in particular, too flexible to guarantee the existence of an equilibrium), we impose three assumptions. These assumptions, referred to as Exchangeability, Monotonicity, and Centeredness, actually impose some structure on the multi-battle contest. Indeed, as our first main result shows, any contest that satisfies our assumptions decomposes naturally into two phases. In the principal phase that is entered into at the start of the contest, every state is visited at most once. In the subsequent tie-breaking phase, however, every non-terminal state 
may be revisited any number of times. Multi-battle contests with finite horizon, such as the match race, are degenerate in the sense that there is no tie-breaking phase. Another class of degenerate examples is composed of tug-of-wars that begin directly with the tie-breaking phase. In fact, it turns out that, under the assumptions that we impose, any tie-breaking phase is "isomorphic" to a tug-of-war.

Given the salience of the tug-of-war in our characterization result, we consider this particular type of dynamic contest in some detail. Assuming a battle technology with constant or declining returns, it is shown that the tug-of-war admits a unique symmetric Markov perfect equilibrium, which is necessarily interior. We also characterize the equilibrium. While the endogenous quantities such as strategies, conditional winning probabilities, and expected continuation payoffs may be represented in explicit form only in special cases, we identify a general (and quite useful) similarity principle of the Markov perfect equilibrium. This property says that the continuation payoffs in a tug-of-war with any given number of states can be easily derived from the continuation values in a tug-of-war with more states.

More generally, we prove the existence of a unique symmetric Markov perfect equilibrium for any multi-battle contest that satisfies our assumptions. Provided that the contest has a finite horizon, it is of course the standard backward induction argument that is used to determine equilibrium efforts. The case of contests with a potentially infinite horizon is more complicated. Based upon the structural description of our multi-battle contests, however, the problem reduces to finding an equilibrium in the tie-breaking phase. Since we know that this is always a tug-of-war, we may build on our previously obtained structural results for that case. Thereby, we determine expected payoffs in a large class of dynamic contests with a potentially infinite horizon.

Equipped with the assurance that the Markov perfect equilibrium is unique and with an effective method to compute the equilibrium for any functional assumption on the battle technology, we examine the properties of multi-battle contests from the perspective of a designer that aims at maximizing total expected effort in the multi-battle contest (i.e., across players and across stages, and without discounting). Blending our limited theoretical results with findings from extensive numerical analysis, we arrive at four main conclusions. First, the one-shot contest is actually worse than any dynamic contest. Second, the optimal unrestricted multi-battle contest is always a match race. Third, the length of the optimal race is monotonically declining in the decisiveness of the contest technology. Fourth, if complexity is costly and there is sufficient noise, then the tug-of-war tends to dominate any other contest. 


\subsection{Related literature}

For an introduction to dynamic contests, see Konrad (2009, Ch. 8) or Szymanski (2003, Sec. 2.6). Following the ground-breaking work by Fudenberg et al. (1983), a key contribution to the literature has been Harris and Vickers (1987) who studied the match race and the tug-of-war assuming that each battle is a probabilistic contest as in Lee and Wilde (1980). They showed existence of a Markov Perfect Equilibrium for the tug-of-war, and also offer conditions sufficient for uniqueness. ${ }^{1}$ Below, we build on their insights, yet using a different contest technology. Grossman and Shapiro (1987, first draft 1985) considered a related model of a race with uncertainty, yet restricted attention to the case of twostages. Judd (2003, likewise, first draft 1985) offers an interesting model of a multi-stage race in which stochastic progress is measured on a continuous scale. Taylor (1995) allows for incomplete information in a dynamic research tournament.

After this initial period, both the match race and the tug-of-war have been studied quite thoroughly under the assumption of a deterministic contest technology. In this case, tie-breaking rules may matter for the equilibrium outcome. Stipulating that the player with the positive continuation value wins, Konrad and Kovenock (2005) identified an intuitive equilibrium outcome in which positive levels of effort are expended only at the initial state. In contrast, Agastya and McAfee (2006), see also McAfee (2000), assumed that a state is revisited if both efforts are zero, and showed that a stalemate of this type may occur in a Markov perfect equilibrium. In an applied variant of the tug-of-war, McBride and Skaperdas (2006) assumed that an additional bias supports the initial victor. Fu et al. (2015) point out that dynamic team contests behave entirely different than contests between individuals. Häfner (2017) considered a tug-of-war between groups, where in each stage, a pair of agents is matched under incomplete information. He finds a unique Markov-perfect equilibrium with interesting comparative statics. Karagözoglu et al. (2018) were first in proving existence and uniqueness of a Markov perfect equilibrium of a tug-of-war with a lottery technology and quadratic costs, which corresponds in our setting to the special case of a Tullock contest with parameter $R=\frac{1}{2} \cdot{ }^{2}$ Correspondingly, our Theorem 2, including also the similarity principle for continuation payoffs, extends their equilibrium characterization

\footnotetext{
${ }^{1}$ See also the working paper version (Harris and Vickers, 1986), as well as the stochastic-process difference-form formulation of the tug-of-war (Budd et al., 1993).

${ }^{2}$ Karagözoglu et al. (2018) multiply the first-order conditions with each other and solve for the equilibrium effort. As they note, however, that trick works only for the case of quadratic costs. Karagözoglu et al. (2018, p. 22) conclude: "Whether our results generalize to an impact function of the form, $f(x)=x^{r}$, where $r<1$ or $1<r \leq 2$ is far from trivial, and hence left as an open question." Since their $r$ corresponds to $2 R$ in our notation, Section 4 of the present paper addresses precisely this open research question.
} 
in a number of ways. Klumpp and Polborn (2006) considered races with Tullock technology. Assuming a deterministic contest technology, Konrad and Kovenock (2009) studied races with heterogeneous deadlines, a potential headstart for one player, and positive intermediate prizes. Multi-player races have been considered by Doğan et al. (2018). None of these contributions, however, uses finite automata to define a flexible class of dynamic multi-battle contest structures. Neither does any of these contributions characterize the Markov perfect equilibrium in the tug-of-war for a general class of contest technologies. ${ }^{3}$

In our setting, the effort provision is the lower the more unbalanced the state. This is in line with contest models in the tradition of Dixit (1987), for instance. However, in a framework with infinitely many stages and no premature end, Hörner (2004) shows that the leader in a highly unbalanced state may exert excessive effort so as to keep the follower at a safe distance.

The type of finite state machine introduced below is known as Moore (1956) machines. Rubinstein (1986) used finite automata to model the strategy choice of boundedly rational players in infinitely repeated non-cooperative games. Salant (2011) employed finite automata to model boundedly rational choice. In contrast to those papers, we use the Moore machine to model a system of rules for a multibattle contest.

\subsection{Overview over the paper}

The remainder of this paper is structured as follows. Multi-battle contests are introduced in Section 2. Section 3 presents the characterization result for multi-battle contests that satisfy our definitions. The tug-of-war is discussed in Section 4. Section 5 deals with the existence of a unique Markov perfect equilibrium. Revenue-maximizing contests are studied in Section 6. Section 7 concludes. The analysis of the tug-of-war and other technical derivations have been relegated to an Appendix.

\section{Multi-battle contests}

\subsection{Finite state machines}

Two risk-neutral contestants $i \in\{1,2\}$ repeatedly face each other in the same type of component contest (or battle), until the rules of the game determine that the contest ends, in which case one of the players is declared the winner and the other is declared the loser. The winner of the multi-battle contest receives

\footnotetext{
${ }^{3}$ Dynamic contests are nice objects for experimental analysis as well. Zizzo (2002) tests the race (best-of-19) in the lab. Deck and Sheremata (2015) offer an experimental analysis of the tug-of-war with all-pay technology. Mago and Sheremeta (2017) implement the race (best-of-three) as an experiment with the all-pay auction as CSF. Dechenaux et al. (2015) survey the literature.
} 
a prize of value $V>0$, while the loser obtains no prize. Without loss of generality, the prize will be normalized to one, i.e., $V=1$. As mentioned in the Introduction, we will consider multi-battle contest rules that may be expressed in terms of a finite deterministic state machine.

Definition 1. A multi-battle contest $\mathcal{M}$ is composed of:

(i) a finite set of states $\Omega$, including an initial state $\omega^{\text {init }} \in \Omega$;

(ii) a partition $\Omega=\Omega_{\text {act }} \cup \Omega_{1} \cup \Omega_{2}$ of $\Omega$ into three pairwise disjoint and nonempty subsets $\Omega_{\text {act }}, \Omega_{1}$, and $\Omega_{2} ;$ and

(iii) a transition map $\alpha: \Omega_{\text {act }} \times\{1,2\} \rightarrow \Omega$.

Any state $\omega \in \Omega_{\text {act }}$ will be referred to as an active state, and any state $\omega \in \Omega_{i}$, with $i \in\{1,2\}$, will be called a winning state for player $i$. The interpretation is that the multi-battle contest starts (i.e., the machine begins to run) at time $t=1$ at state $\omega_{1}=\omega^{\text {init }}$. Then, for any $t \geq 1$ such that $\omega_{t} \in \Omega_{\text {act }}$, there is a battle at time $t$. Depending on the winner $i_{t} \in\{1,2\}$ of the battle at time $t$, the machine transits into a new state $\omega_{t+1}=\alpha\left(\omega_{t}, i_{t}\right)$. The multi-battle contest ends (i.e., the machine stops) when $\omega_{t} \in \Omega_{i}$ for some $i \in\{1,2\}$. Then, player $i$ (player $j \in\{1,2\}, j \neq i$ ) is declared the winner (the loser) of the multi-battle contest.

The following two examples illustrate the definition.

Example 1 ("Match race" or "Win-with- $M$ "). Let $M \geq 1$ be an integer. In a match race of length $M$, the set of active states is given by $\Omega_{\text {act }}=\left\{\omega^{\left(m_{1}, m_{2}\right)}: m_{1}, m_{2} \in\{0, \ldots, M-1\}\right\}$, and the initial state by $\omega^{\text {init }}=\omega^{(0,0)}$. Terminal states are collected in sets $\Omega_{1}=\left\{\omega^{\left(M, m_{2}\right)}: m_{2} \in\{0, \ldots, M-1\}\right\}$, and $\Omega_{2}=$ $\left\{\omega^{\left(m_{1}, M\right)}: m_{1} \in\{0, \ldots, M-1\}\right\}$. Further, the transition map is given by $\alpha\left(\omega^{\left(m_{1}, m_{2}\right)}, 1\right)=\omega^{\left(m_{1}+1, m_{2}\right)}$ and $\alpha\left(\omega^{\left(m_{1}, m_{2}\right)}, 2\right)=\omega^{\left(m_{1}, m_{2}+1\right)}$, respectively, where $m_{1}, m_{2} \in\{0, \ldots, M-1\}$.

Example 2 ("Tug-of-War" or "Win-by- $N$ "). Let $N \geq 1$ be an integer. In a tug-of-war of (equi-) distance $N$, or with $(2 N-1)$ active states, it is specified that $\Omega_{\text {act }}=\left\{\omega^{-(N-1)}, \ldots, \omega^{N-1}\right\}, \omega^{\text {init }}=\omega^{0}$, $\Omega_{1}=\left\{\omega^{N}\right\}$, and $\Omega_{2}=\left\{\omega^{-N}\right\}$. In this case, the transition map is given by $\alpha\left(\omega^{n}, 1\right)=\omega^{n+1}$ and $\alpha\left(\omega^{n}, 2\right)=\omega^{n-1}$, for any $n \in\{-(N-1), \ldots, N-1\}$.

Thus, in a match race of length $M$, a player having won $M$ battles wins the multi-battle contest, where the improper case $M=1$ corresponds to the one-shot contest. We will refer to a race of length $M$ alternatively as a win-with-M. It is easy to see that the maximum number of consecutive battles in 
a race of length $M$ is $(2 M-1)$. Therefore, for instance, a race of length $M=2$ (of length $M=3$ ) corresponds to what is known in the literature as a best-of-three (best-of-five) multi-battle contest. In the tug-of-war of distance $N$, however, a player needs to win $N$ battles more than the opponent. Again, the improper case $N=1$ corresponds to the one-shot contest. Note that, for $N \geq 2$, the tug-of-war has a potentially infinite horizon. We will refer to a tug-of-war of distance $N$ alternatively as a win-by- $N$.

\subsection{Symmetric and minimal contests}

It is not difficult to see that two distinct automata may describe multi-battle contests that are "isomorphic" for the contestants. For example, there could be states in the Moore machine that are never reached in finite play. Even if all states are reachable, there may be redundancies in the representation. To make this precise, we introduce the following definitions. Given a multi-battle contest $\mathcal{M}$, let $H_{i}(\mathcal{M})$ denote the set of finite histories for each player $i \in\{1,2\}$ that end in a state $\omega^{T+1} \in \Omega_{i}$. We say that two multi-battle contests $\mathcal{M}$ and $\mathcal{M}^{\prime}$ are equivalent $\left(\mathcal{M} \sim \mathcal{M}^{\prime}\right.$ in short) if $H_{1}(\mathcal{M})=H_{1}\left(\mathcal{M}^{\prime}\right)$ and $H_{2}(\mathcal{M})=H_{2}\left(\mathcal{M}^{\prime}\right)$.

For a given multi-battle contest $\mathcal{M}$ with state space $\Omega$, we call the number of elements of $\Omega$ the complexity $\gamma(\mathcal{M})$ of $\mathcal{M}$. Note that the complexity counts not only active, but also terminal states. For example, the win-with-two (i.e., a race of length two) has a complexity of eight states, because there are four active and four terminal states. Clearly, by merging terminal states, an equivalent contest can be found with no more than $\gamma=6$ states. This motivates the following definition.

Definition 2. A multi-battle contest $\mathcal{M}$ is minimal if, for any multi-battle contest $\mathcal{M}^{\prime}$ equivalent to $\mathcal{M}$, we have $\gamma(\mathcal{M}) \leq \gamma\left(\mathcal{M}^{\prime}\right)$.

Assumption 1. The contest is minimal.

This assumption will be tacitly used in the proofs. However, in our illustrations of specific contests, it proves useful to work with non-minimal contests. Obviously, Assumption 1 is not a limitation at all in the sense because it is not structural but only about the representation of contests. Note also that, in a minimal contest $\mathcal{M}$ in which both contestants have the opportunity to win, the number of active states equals $\gamma(\mathcal{M})-2$.

We will focus on multi-battle contests that are ex-ante symmetric for the players.

Definition 3. A multi-battle contest $\mathcal{M}$ is called symmetric if there is a bijection $\pi: \Omega \rightarrow \Omega$ such 
that (i) $\pi\left(\omega^{\text {init }}\right)=\omega^{\text {init }}$, (ii) $\pi$ induces a bijection from $\Omega_{1}$ onto $\Omega_{2}$, and (iii) $\pi(\alpha(\omega, i))=\alpha(\pi(\omega), j$ ) for any $\omega \in \Omega_{\text {act }}$ and $i, j \in\{1,2\}$ with $j \neq i$.

The interpretation of the symmetry condition is straightforward. Since, by Assumption 1, every state is reachable from the initial state $\omega^{\text {init }}$, a straightforward induction argument may be used to show that, if a multi-battle contest is symmetric, then the bijection $\pi$ is unique. We will call a state $\omega \in \Omega$ balanced if $\pi(\omega)=\omega$.

For instance, in Example 1, the mapping $\pi$ maps any state $\omega^{\left(m_{1}, m_{2}\right)} \in \Omega$ to $\omega^{\left(m_{2}, m_{1}\right)}$. In particular, a state $\omega^{\left(m_{1}, m_{2}\right)}$ is balanced if and only if $m_{1}=m_{2} \in\{0, \ldots, M-1\}$. Similarly, in Example 2, the mapping $\pi$ maps any state $\omega^{n} \in \Omega$ to $\omega^{-n}$. Moreover, state $\omega^{n}$ is balanced if and only if $n=0$.

Assumption 2. The contest is symmetric.

\subsection{Component contests}

Given effort levels $x_{1} \geq 0$ for player 1 and $x_{2} \geq 0$ for player 2 , consider a battle with stage payoffs

$$
\begin{aligned}
& \Pi_{1}\left(x_{1}, x_{2}\right)=p\left(x_{1}, x_{2}\right) V_{1}-x_{1} \\
& \Pi_{2}\left(x_{1}, x_{2}\right)=\left(1-p\left(x_{1}, x_{2}\right)\right) V_{2}-x_{2},
\end{aligned}
$$

where $V_{1}$ and $V_{2}$, respectively, are player 1 's and player 2' valuations, and $p: \mathbb{R}_{+} \times \mathbb{R}_{+} \rightarrow[0,1]$ is the contest success function (CSF), capturing the probability that contestant 1 wins the battle. Constant unit costs are assumed for expositional simplicity. Indeed, as usual, the consideration of continuously differentiable, convex and strictly increasing cost functions that vanish at zero effort can be accomplished by a simple transformation of the effort variable.

A CSF is called concave if $p\left(\cdot, x_{2}\right)$ is concave on $\mathbb{R}_{+}$, for any $x_{2} \geq 0$; and strictly concave (in the interior) if $p\left(\cdot, x_{2}\right)$ is strictly concave on $\mathbb{R}_{+}$, for any $x_{2}>0$. The CSF $p$ is strictly monotone (in the interior) if $p\left(\widehat{x}_{1}, x_{2}\right)>p\left(x_{1}, x_{2}\right)$, for any $\widehat{x}_{1}>x_{1} \geq 0$, and $x_{2}>0$; anonymous if $p\left(x_{2}, x_{1}\right)=1-p\left(x_{1}, x_{2}\right)$, for any $x_{1} \geq 0$ and $x_{2} \geq 0$; differentiable (in the interior) if $\partial p\left(x_{1}, x_{2}\right) / \partial x_{1}$ exists and is continuous for any $x_{1} \geq 0$ and $x_{2}>0$; competitive if $p\left(0, x_{2}\right)=0$ for any $x_{2}>0$; homogeneous (of degree zero) if $p\left(\lambda x_{1}, \lambda x_{2}\right)=p\left(x_{1}, x_{2}\right)$ for any $\lambda>0, x_{1} \geq 0$, and $x_{2} \geq 0$. Following the elegant treatment of Baik (2004), Malueg and Yates (2005, 2006), amongst others, we will impose the following assumption.

Assumption 3. The component CSF is concave, strictly monotone, anonymous, continuously differentiable, competitive, and homogeneous. 
Define auxiliary functions $f(y)=p(y, 1)$ and $\phi(y)=f(y)-y f^{\prime}(y)$. Then, $f$ is strictly increasing, twice differentiable, and concave, and satisfies the functional equation $f(1 / y)=1-f(y){ }^{4}$ Moreover, $\phi(\cdot)$ is continuous and strictly increasing, with $\phi(0)=0$ and $\sigma \equiv \phi(1) \in\left(0, \frac{1}{2}\right)$.

We may express equilibrium efforts and payoffs in terms of the CSF as follows.

Lemma 1. Impose Assumption 3. Then the probabilistic contest admits a pure-strategy Nash equilibrium if and only if either (i) $V_{1}>0$ and $V_{2}>0$, or (ii) $V_{1} \leq 0$ and $V_{2} \leq 0$. If the equilibrium exists, it is unique. In case (i), equilibrium effort levels are given by

$$
x_{1}^{*}=V_{2} f^{\prime}\left(\frac{V_{2}}{V_{1}}\right) \text { and } x_{2}^{*}=V_{1} f^{\prime}\left(\frac{V_{1}}{V_{2}}\right)
$$

and equilibrium payoffs are given by

$$
\Pi_{1}^{*}=V_{1} \phi\left(\frac{V_{1}}{V_{2}}\right) \text { and } \Pi_{2}^{*}=V_{2} \phi\left(\frac{V_{2}}{V_{1}}\right) .
$$

In particular, equilibrium payoffs are positive. In case (ii), $x_{1}^{*}=x_{2}^{*}=0, \Pi_{1}^{*}=V_{1} / 2$ and $\Pi_{2}^{*}=V_{2} / 2$.

There is a wide variety of probabilistic specifications that are consistent with Assumption 3. Some examples are listed below.

Tullock CSF. Following Tullock (1980), one may assume that player 1's probability of winning is given as $p^{\mathrm{TUL}}\left(x_{1}, x_{2}\right)=x_{1}^{R} /\left(x_{1}^{R}+x_{2}^{R}\right)$ for some $R \in(0,1]$, with the convention that the ratio is read as $1 / 2$ if $x_{1}=x_{2}=0$. For the Tullock case, $f^{\mathrm{TUL}}(\xi)=\frac{\xi^{R}}{1+\xi^{R}}$ with derivative $f^{\prime \mathrm{TUL}}(\xi)=\frac{R \xi^{R-1}}{\left(1+\xi^{R}\right)^{2}}$, and $\phi^{\mathrm{TUL}}(\xi)=\frac{\xi^{R}\left(\xi^{R}+1-R\right)}{\left(1+\xi^{R}\right)^{2}}$. For $R=1$, this CSF is a simple lottery. ${ }^{5}$

Serial CSF. Let $\alpha \in(0,1)$. Player 1's probability of winning is given as $p^{\mathrm{SER}}\left(x_{1}, x_{2}\right)=1-\frac{1}{2}\left(x_{2} / x_{1}\right)^{\alpha}$ if $x_{1} \geq x_{2}$, and by $p^{\mathrm{SER}}\left(x_{1}, x_{2}\right)=\frac{1}{2}\left(x_{1} / x_{2}\right)^{\alpha}$ if $x_{1}<x_{2}$. Again, we have the convention that the ratio $x_{1} / x_{2}$ is read as $1 / 2$ if $x_{1}=x_{2}=0$. This specification has been introduced by Alcalde and Dahm (2007).

Relative-difference CSF. Finally, let $p^{\mathrm{RD}}\left(x_{1}, x_{2}\right)=\frac{(1+\tau) x_{1}+(1-\tau) x_{2}}{2\left(x_{1}+x_{2}\right)}$, where $\tau \in(0,1]$. This specification is a special case of a functional form introduced by Beviá and Corchón (2015). It corresponds to a

\footnotetext{
${ }^{4}$ Indeed,
}

$$
f\left(\frac{1}{x}\right)=p\left(\frac{1}{x}, 1\right)=p(1, x)=1-p(x, 1)=1-f(x) .
$$

${ }^{5}$ The case $R \in(1,2)$ may lead to battles in which one of the players randomizes, while the case $R \geq 2$ renders each battle equivalent to an all-pay auction. In both cases, one of the two players ends up with a zero expected payoffs, which leads to strategic considerations covered, in essence, already by Agastia and McAfee (2006) and Konrad and Kovenock (2005). 
convex combination of a Tullock contest with parameter $R=1$, which receives a weight of $\tau$, and a coin toss, which receives a weight of $1-\tau$.

However, the differentiability condition in Assumption 3 excludes the all-pay auction, which has been studied before by Agastya and McAfee (2006) and Konrad and Kovenock (2005). The homogeneity assumption excludes lotteries with noise, as studied by Blavatskyy (2010), for instance. Homogeneity is crucial for our analysis of the tug-or-war, so we have presently no way of including such cases, even though it seems plausible that our results should extend. The main reason for Assumption 3 is that we can thereby ensure existence of a unique pure-strategy Nash equilibrium.

\subsection{Histories, strategies, and Markov perfect equilibrium}

By a finite history $h$, we mean a finite sequence $\left(i_{1}, i_{2}, \ldots, i_{T}\right)$ of players in $\{1,2\}$ (where $i_{t}$ is interpreted as the winner of the $t$-th battle), such that there is a finite sequence $\left(\omega_{1}, \omega_{2}, \ldots, \omega_{T+1}\right)$ of states in $\Omega$ satisfying (i) $\omega_{1}=\omega^{\text {init }}$, and (ii) $\omega_{t} \in \Omega_{\text {act }}$ with $\omega_{t+1}=\alpha\left(\omega_{t}, i_{t}\right)$, for any $t \in\{1, \ldots, T\}$. In this case, we will say that $h$ is of length $T \geq 0$ and ends in state $\omega^{T+1}$. Denote by $H$ the set of all finite histories $h$. Similarly, an infinite history $h$ consists of a sequence $\left\{i_{t}\right\}_{t=1}^{\infty}$, such that there is a sequence $\left\{\omega_{t}\right\}_{t=1}^{\infty}$ satisfying (i) $\omega_{1}=\omega^{\text {init }}$, and (ii) $\omega_{t} \in \Omega_{\text {act }}$ with $\omega_{t+1}=\alpha\left(\omega_{t}, i_{t}\right)$, for any $t \in \mathbb{N} \equiv\{1,2, \ldots\}$. A terminal history $h$ is either a finite history of length $T$ such that $\omega_{T+1} \in \Omega_{1} \cup \Omega_{2}$, or an infinite history. A finite history $h \in H$ given by finite sequences $\left(i_{1}, i_{2}, \ldots, i_{T}\right)$ is a subhistory of some history $h^{\prime}$ of length $T^{\prime} \in\{T, T+1, \ldots\} \cup\{\infty\}$ given by (finite or infinite) sequences $\left(i_{1}^{\prime}, i_{2}^{\prime}, \ldots\right)$ if $i_{t}=i_{t}^{\prime}$ for any $t \in\{1, \ldots, T\}$. We call a state $\omega \in \Omega$ reachable if there is a finite history $h \in H$ ending in $\omega=\omega_{T+1}$. A recurrent state $\omega \in \Omega$ is a state that can be visited more than once during play.

Let $H_{\text {act }} \subseteq H$ denote the set of histories $h \in H$ that end in an active state $\omega_{T+1} \in \Omega_{\text {act }}$. A pure strategy $\xi_{i}: H_{\text {act }} \rightarrow \mathbb{R}_{+} \equiv[0, \infty)$ for player $i$ specifies an action $x_{i}=\xi_{i}(h) \geq 0$ for any $h \in H_{\text {act }}$. We denote by $S_{i}$ the set of pure strategies for contestant $i \in\{1,2\}$. Given a finite history $h \in H$, we consider the set $H_{\text {term }}(h)$ of terminal histories that have $h$ as a subhistory. Then, given a pair of pure strategies $\left(\xi_{1}, \xi_{2}\right) \in S_{1} \times S_{2}$, and given a contest success function $\left\{p_{i}(\cdot, \cdot)\right\}_{i=1,2}$, we may define the outcome of the game as the probability distribution over $H_{\text {term }}(h)$ induced by $\left(\xi_{1}, \xi_{2}\right){ }^{6}$

\footnotetext{
${ }^{6}$ While $H_{\text {term }}(h)$ is an infinite set, there are no hidden technical obstacles here. To understand why, note that $H_{\text {term }}(h)$ may be understood as a projection of the product space $\{1,2\}^{\mathbb{N}}$, where we identify two points if they go through the same terminal state. Since the projection is surjective, we obtain a sigma field on $H_{\text {term }}(h)$ in a natural way, and may consider the push-forward measure on the image space $H_{\text {term }}(h)$.
} 
Given a finite terminal history and a finite sequence of effort choice

$$
\left(\left(x_{1}^{1}, x_{2}^{1}\right), \ldots,\left(x_{1}^{T}, x_{2}^{T}\right)\right) \in \mathbb{R}_{+}^{2 T},
$$

we assign payoff $1-\sum_{t=1}^{T} x_{t}^{i}$ to a multi-battle contest-winning player $i$, and similarly, $-\sum_{t=1}^{T} x_{t}^{i}$ to a multi-battle contest-loser player $i$, at any finite terminal history of length $T$. Further, payoff $\frac{1}{2}-\sum_{t=1}^{\infty} x_{t}^{i}$ is assigned to player $i$ from any infinite terminal history.

Given a finite history $h \in H$, and a pair of pure strategies $\left(\xi_{1}, \xi_{2}\right)$, player $i$ 's continuation payoff at $h$ is the expectation of terminal payoffs over the resulting outcome. ${ }^{7}$ A pair of pure strategies $\left(\xi_{1}, \xi_{2}\right)$ is a subgame-perfect equilibrium if, for any finite history $h$ ending in $\omega_{T+1} \in \Omega_{\text {act }}$, each player $i \in\{1,2\}$ maximizes her continuation payoff at $\omega_{T+1}$ by choosing $\xi_{i}(h)$. A pure strategy is Markovian if $\xi_{i}(h)$ may depend on the finite history $h$ only through the most recent state $\omega_{T+1}$ (Ericson and Pakes, 1995; Maskin and Tirole, 2001). A subgame-perfect equilibrium is a Markov perfect equilibrium (MPE) when it employs Markovian strategies. We call an MPE interior if players exert a positive effort at all active states.

\section{Characterization result}

\subsection{Assumptions}

It turns out that, for general multi-battle contests, a MPE need not exist (for examples, see Section 5).

To avoid this non-existence problem, and also because we had the impression that not all multi-battle contests make sense (i.e., the definition appeared to us as being still too flexible), we will impose the following three assumptions.

Assumption (E for Exchangeability). For any $\omega \in \Omega_{\text {act }}$ such that $\omega^{\prime} \equiv \alpha(\omega, 1) \in \Omega_{\text {act }}$ and $\omega^{\prime \prime} \equiv \alpha(\omega, 2) \in \Omega_{\text {act }}$, we have $\alpha\left(\omega^{\prime}, 2\right)=\alpha\left(\omega^{\prime \prime}, 1\right)$.

Assumption (M for Monotonicity). For any $\omega \in \Omega_{\text {act }}$ and $i \in\{1,2\}$, there is a finite sequence $\omega^{1}, \ldots, \omega^{L}$ with $L \geq 1$ such that (i) $\omega^{1}=\omega$, (ii) $\omega^{l} \in \Omega_{\text {act }}$ with $\omega^{l+1}=\alpha\left(\omega^{l}, i\right)$, for $l \in\{1, \ldots, L-1\}$, and (iii) $\omega^{L} \in \Omega_{i}$.

\footnotetext{
${ }^{7}$ This expected payoff exists in the extended real line $\mathbb{R} \cup\{-\infty\}$ because a contestant's expected revenues are finite. Indeed, a contestant may not collect more prize money than $V=1$. However, since the contest is repeated, and the outcome of each battle uncertain, there is no upper limit to the contestant's total of ex-post delivered efforts. Economically, it should be obvious that no player ever finds it optimal to choose a pure strategy that leads to a negative expected payoff. We may therefore assume, without loss of generality, that the continuation payoff is finite.
} 
Assumption ( $\mathbf{C}$ for Centeredness). There is at most one state $\omega \in \Omega$ that is both recurrent and balanced.

Exchangeability captures the requirement that any two finite histories that differ only in the order in which the last two contests have been won end up in the same state. For instance, Exchangeability would hold within a tennis game, but it would fail to hold in a tennis match considering points as battles. To understand why, suppose that, at a score of 40:15 in the first game of the match, the server makes a point and wins the game, but loses the first point of the next game, so that the score is 1:0 $0: 15$. This is certainly a situation that strategically differs from the one reached if the server first loses and then wins a point, resulting in the score 1:0 0:0. The possibility that a tennis match can be won with less than half of the total points is well-known, of course. ${ }^{8}$

Next, Monotonicity says that, starting from any active state, winning all battles in a row will let you win the multi-battle contest. Monotonicity implies, in particular, that in order to win the multi-battle contest, a player must necessarily win the last battle. We will later see that this assumption is crucial for equilibrium existence. It may also be noted that Monotonicity combined with Exchangeability excludes multi-battle contests in which the outcome is determined by simple majority. Indeed, this would be pointless because the winner may be determined before the last battle. ${ }^{9}$

Finally, Centeredness says that there is at most one balanced state that may be revisited after a sequence of battles. As we show below, both Exchangeability and Monotonicity are indispensable for equilibrium existence. Centeredness, however, does not seem crucial for existence but dropping it leads, as will become clear below, to a less intuitive class of multi-battle contests.

\subsection{Strongly connected components}

A state $\omega \in \Omega$ is called finite if there exists $T^{*}=T^{*}(\omega) \geq 0$ such that, for any finite history $h$ ending in $\omega=\omega^{T+1}$, we have $T \leq T^{*}$ We will say that a multi-battle contest has a finite horizon if each state is finite. Conversely, a multi-battle contest that does not possess a finite horizon will be referred to as having an infinite horizon. A strongly connected component $(S C C)$ is a maximal subset $C \subseteq \Omega_{\text {act }}$ with the properties that (i) $C$ has at least two elements, and (ii) any state $\omega \in C$ can be reached from any other state $\omega^{\prime} \in C$. Clearly, an SCC consists of recurrent states only.

Lemma 2. A multi-battle contest has an infinite horizon if and only if it has an SCC.

\footnotetext{
${ }^{8}$ Wright et al. (2013) estimate that this anomaly occurs in approximately 5 percent of all tennis matches.

${ }^{9}$ This would change, however, if stage prizes are admitted. See Section 7 for discussion.
} 
Proof. Immediate from the definitions.

\subsection{Statement of the result}

The following is the first main result of this paper. It is crucial for our analysis of general multi-battle contest structures.

Theorem 1. Impose Assumptions (E), $(M)$, and $(C)$. Then, either the multi-battle contest has a finite horizon, or there is precisely one multi-battle contest-ending tug-of-war that is reached after a maximum finite number of steps.

Two simple classes of contests that are consistent with the prediction of Theorem 1 are the following.

Example 3 ("Win-with- $M$-or-by- $N$ ") This finite-horizon contest may be thought of as a match race of length $M \geq 2$ that is ended prematurely if one of the contestants has an advantage of at least $N$ points over the opponent. Note that for $N \geq M$, this is equivalent to a race of length $M$. Conversely, if $N=1$, then we have a one-shot contest. Therefore, we will assume that $N \in\{2, \ldots, M-1\}$. The simplest non-trivial case is the win-with-three-or-by-two, which is illustrated in Figure 1(a).

Example 4 ("Win-with- $M$-and-by- $N "$ ) This infinite-horizon contest may be thought of as a match race of length $M \geq 2$ that is continued until one of the contestants has an advantage of at least $N$ points over the opponent. For $N \geq M$, this is equivalent to a tug-of-war of distance $N$. Conversely, if $N=1$, then we have a match race. Therefore, we will assume again that $N \in\{2, \ldots, M-1\}$. The simplest non-trivial case, the win-with-three-and-by-two, is illustrated in Figure 1(b). ${ }^{10}$

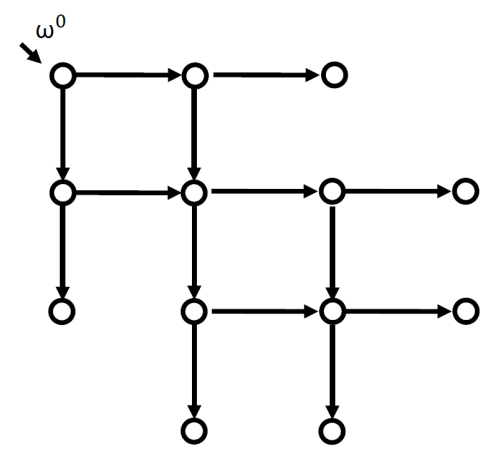

(a)

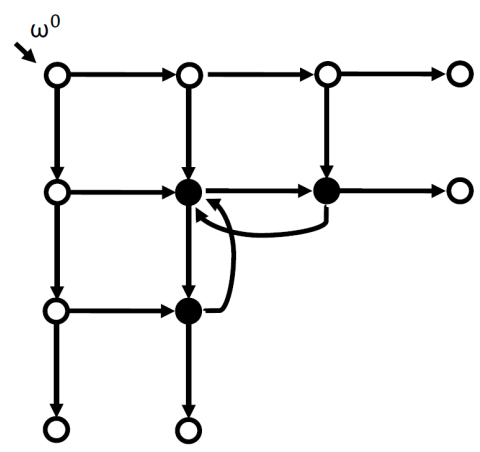

(b)

Figure 1. (a) Win-with-3-or-by-2; (b) Win-with-3-and-by-2

\footnotetext{
${ }^{10} \mathrm{~A}$ another example is the win-with-four-and-by-two, which captures the counting system in a tennis game.
} 
In the remainder of this subsection, we prove Theorem 1. The proof is based on a sequence of lemmas. Suppose first that the multi-battle contest has an infinite horizon. Then, by Lemma 2, there exists an SCC.

For states $\omega, \omega^{\prime} \in \Omega$ and a player $i \in\{1,2\}$ such that $\alpha(\omega, i)=\omega^{\prime}$, we say that the move from $\omega$ to $\omega^{\prime}$ is irreversible if there is no finite sequence $\left(i_{1}, i_{2}, \ldots, i_{T}\right)$ of players in $\{1,2\}$ (where $i_{t}$ is the winner of the $t$-th battle) and a finite sequence $\left(\omega_{1}, \omega_{2}, \ldots, \omega_{T+1}\right)$ of states in $\Omega$ such that (i) $\omega_{1}=\omega^{\prime}$, (ii) $\omega_{T+1}=\omega$, and (iii) $\omega_{t} \in \Omega_{\text {act }}$ with $\omega_{t+1}=\alpha\left(\omega_{t}, i_{t}\right)$, for any $t \in\{1, \ldots, T\}$. The following lemma shows that any irreversible move leading away from any state in an SCC leads to a terminal state.

Lemma 3. Impose Assumptions (E) and (M). Suppose that $\omega \in \Omega$ is recurrent and that the move to $\omega^{\prime} \equiv \alpha(\omega, 1)$ is irreversible. Then $\omega^{\prime}$ is terminal.

Proof. Since $\omega$ is recurrent, it is contained in some SCC $C$ (see Figure 1 for illustration). We claim that $\omega^{\prime}=\alpha(\omega, 1)$ is terminal. Suppose not. Then $\omega^{\prime} \in \Omega^{\text {act }}$. Moreover, since $\omega$ is recurrent, and the move to $\omega^{\prime}$ is irreversible, $\omega^{\prime \prime}=\alpha(\omega, 2) \in C$ must be recurrent. In particular, $\omega^{\prime \prime} \in \Omega_{\text {act }}$. By Exchangeability, $\alpha\left(\omega^{\prime}, 2\right)=\alpha\left(\omega^{\prime \prime}, 1\right) \equiv \omega^{\prime \prime \prime}$. Further, by Monotonicity, a player winning a battle and ending the multi-battle contest must win the multi-battle contest. Hence, it is impossible that $\omega^{\prime \prime \prime}$ is a terminal state. Hence, $\omega^{\prime \prime \prime} \in \Omega^{\text {act }}$. Next, since the move from $\omega$ to $\omega^{\prime}$ is irreversible, so is the move from $\omega$ to $\omega^{\prime \prime \prime}$. But, $\omega$ and $\omega^{\prime \prime}$ lie in the same SCC. Hence, the move from the recurrent state $\omega^{\prime \prime}$ to $\omega^{\prime \prime \prime}=\alpha\left(\omega^{\prime \prime}, 1\right)$ is irreversible. By induction, and the finiteness of the state space, there is an iteration, at which a repeated win for player 2 leads back to $\omega$. This, however, is in conflict with Monotonicity. The claim follows.

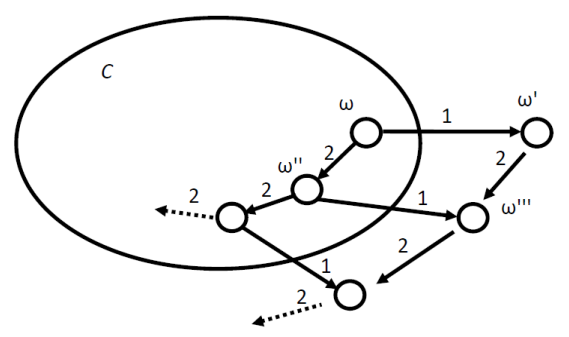

Figure 2. Proof of Lemma 3.

Thus, any state that is reached by an irreversible move from a recurrent state is terminal. To prove the Theorem, it remains to be shown that (i) there cannot be more than one SCC in the multi-battle contest, and (ii) any symmetric SCC is a tug-of-war. 
Lemma 4. Impose Assumptions (E) and (M). Let $C$ be an SCC. Then $C$ can be reached from any active state $\omega \in \Omega_{\mathrm{act}}$. In particular, there is at most one SCC in the multi-battle contest.

Proof. Note that Monotonicity implies that $\omega^{0} \in \Omega_{\text {act }}$. To provoke a contradiction, suppose that there is some active state $\omega \in \Omega_{\text {act }}$ such that $C$ cannot be reached from $\omega$. By Assumption 1, however, $\omega$ can be reached from $\omega^{0}$, i.e., there is a finite sequence of states $\omega^{1}, \ldots, \omega^{K}$ and of players $i^{0}, . ., i^{K-1} \in\{1,2\}$ such that (i) $\omega^{k} \in \Omega_{\text {act }}$ for $k=1, \ldots, K-1$, (ii) $\omega^{k+1}=\alpha\left(\omega^{k}, i^{k}\right)$ for $k=0, \ldots, K-1$, and (iii) $\omega^{K}=\omega$. Since $C$ can be reached from $\omega^{0}$, but cannot be reached from $\omega$, there must be some index $k \in\{0, \ldots, K-1\}$ such that $C$ can be reached from $\omega^{k}$ but not from $\omega^{k+1}$. Without loss of generality, $i^{k}=1$. Since $\omega^{k} \in \Omega_{\mathrm{act}}$, and invoking Monotonicity, there is a finite sequence $\widehat{\omega}^{1}, \ldots, \widehat{\omega}^{L}$ with $L \geq 1$ such that (i) $\widehat{\omega}^{1}=\omega^{k}$, (ii) $\widehat{\omega}^{l+1}=\alpha\left(\widehat{\omega}^{l}, 2\right)$ for $l \in\{1, \ldots, L-1\}$, and (iii) $\widehat{\omega}^{L} \in \Omega_{2}$. Note now that $\omega^{k+1}=\alpha\left(\omega^{k}, 1\right) \in \Omega_{\mathrm{act}}$. If $\alpha\left(\omega^{k}, 2\right)$ is terminal, then we have a contradiction (because there is no SCC at all reachable from $\left.\omega^{k}\right)$. Therefore, $\widehat{\omega}^{2}=\alpha\left(\omega^{k}, 2\right)$ is active, and we have by Exchangeability a state $\alpha\left(\widehat{\omega}^{2}, 1\right)$. By Monotonicity, the state $\alpha\left(\widehat{\omega}^{2}, 1\right)$ is active. From $\alpha\left(\widehat{\omega}^{2}, 1\right)$, we cannot reach $C$, because this would imply we can reach $C$ from $\omega^{k+1}$, which is not true. However, we can reach $C$ from $\widehat{\omega}^{2}$. By induction, we see that only repeated battle wins by player 2 can bring us from $\omega^{k}$ to $C$, and that just one battle win of player 1 on the way leads us to states from which $C$ cannot be reached anymore. But this means that any path in $C$ consists of wins of player 2 exclusively. However, this is inconsistent with Monotonicity! This proves the first assertion. The second assertion follows directly from the first using Lemma 3.

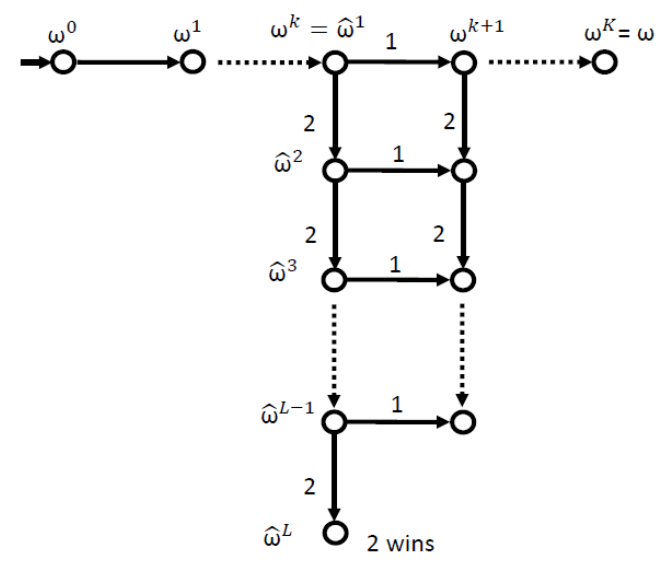

Figure 3. Proof of Lemma 4.

The following lemma, which crucially exploits the symmetry of the contest, identifies an important 
structural property of the tie-breaking phase.

Lemma 5. Impose Assumptions ( $E)$ and $(M)$. Then any SCC contains a balanced state. If, in addition, Assumption (C) holds, then the SCC is a tug-of-war.

Proof. Recall that $\omega^{0}$ has the property that $\pi\left(\omega^{0}\right)=\omega^{0}$. If $\alpha\left(\omega^{0}, 1\right)$ is terminal, then there is no SCC, and we are done. Therefore, $\alpha\left(\omega^{0}, 1\right)$ is active. By Symmetry, the same is true for $\alpha\left(\omega^{0}, 2\right)$. Therefore, by Exchangeability, $\omega^{1} \equiv \alpha\left(\alpha\left(\omega^{0}, 2\right), 1\right)=\alpha\left(\alpha\left(\omega^{0}, 1\right), 2\right)$. By monotonicity, $\omega^{1}$ is active. Moreover, $\pi\left(\omega^{1}\right)=\omega^{1}$. If $\alpha\left(\omega^{1}, 1\right)$ is terminal, then there is no SCC, and we are done (indeed, if $\alpha\left(\omega^{1}, 1\right)$ is terminal, then $\alpha\left(\alpha\left(\omega^{0}, 1\right), 1\right)$ is terminal by Monotonicity. By a symmetry argument, $\alpha\left(\omega^{1}, 2\right)$ is terminal as well and hence again, $\alpha\left(\alpha\left(\omega^{0}, 2\right), 2\right)$ is terminal by Monotonicity. This shows that all paths lead to a terminal state in finitely many steps, i.e., there indeed exists no SCC in this case). Thus, $\alpha\left(\omega^{1}, 1\right)$ is active. By Symmetry, the same is true for $\alpha\left(\omega^{1}, 2\right)$. Therefore, by Exchangeability, $\omega^{2} \equiv \alpha\left(\alpha\left(\omega^{1}, 2\right), 1\right)=\alpha\left(\alpha\left(\omega^{1}, 1\right), 2\right)$. By monotonicity, $\omega^{2}$ is active.

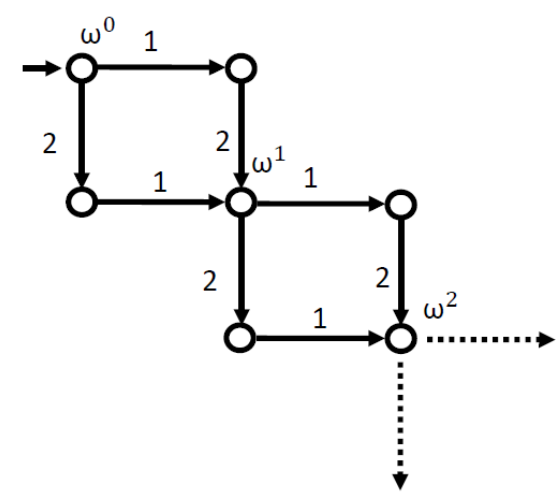

Figure 4. Proof of Theorem 2

Repeating this argument, we have an infinite sequence of active states $\left\{\omega^{k}\right\}_{k=0}^{\infty}$ such that $\pi\left(\omega^{k}\right)=\omega^{k}$ for any $k$. Since the state machine has only finitely many states, there is a state $\omega^{k}$ in that sequence that belongs to the SCC. This proves the first assertion of the theorem.

Assume next that there is precisely one state $\omega \in C$ such that $\pi(\omega)=\omega$. Clearly, $\omega \in \Omega_{\text {act }}$. By Monotonicity, there is a finite sequence $\omega^{1}, \ldots, \omega^{L}$ with $L \geq 1$ such that (i) $\omega^{1}=\omega$, (ii) $\omega^{l} \in \Omega_{\text {act }}$ with $\omega^{l+1}=\alpha\left(\omega^{l}, 1\right)$ for $l \in\{1, \ldots, L-1\}$, and (iii) $\omega^{L} \in \Omega_{1}$. Moreover, there is a finite sequence $\widehat{\omega}^{1}, \ldots, \widehat{\omega}^{K}$ with $K \geq 1$ such that (i) $\widehat{\omega}^{1}=\omega$, (ii) $\omega^{k} \in \Omega_{\text {act }}$ with $\widehat{\omega}^{k+1}=\alpha\left(\widehat{\omega}^{k}, 2\right)$ for $k \in\{1, \ldots, K-1\}$, and (iii) $\omega^{K} \in \Omega_{2}$. By Symmetry, $L=K$, and $\pi\left(\omega^{2}\right)=\widehat{\omega}^{2}$. If $L=2$, then any immediate successor of $\omega$ is terminal, and we obtain a contradiction. Thus, $L>2$. In this case, however, $\omega^{2}$ and $\widehat{\omega}^{2}$ are 
active, and Exchangeability implies that $\alpha\left(\omega^{2}, 2\right)=\alpha\left(\widehat{\omega}^{2}, 1\right)$. Note that this state is balanced, so that $\alpha\left(\omega^{2}, 2\right)=\alpha\left(\widehat{\omega}^{2}, 1\right)=\omega$. Thus, if $L=3$, then we are done. If $L>3$, then we can iteratively use Exchangeability to see that $\alpha\left(\omega^{l+1}, 2\right)=\omega^{l}$ and that $\alpha\left(\widehat{\omega}^{k+1}, 2\right)=\widehat{\omega}^{k}$. This proves the second assertion, and hence, the lemma.

This concludes the proof of Theorem 1.

\subsection{Tightness}

We conclude this section with two examples that illustrate the tightness of our assumptions.

Example 5. The multi-battle contest shown in Figure 5 below satisfies Exchangeability and Monotonicity. Lemma 5 shows, however, that the SCC appearing in Figure 5 cannot be a SCC. This SCC is actually symmetric, but it does not contain a balanced state. Thus, Assumption 1 (symmetry) is crucial for obtaining the conclusion of Lemma 5 .

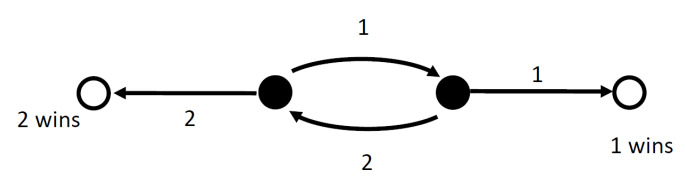

Figure 5. An SCC without balanced state.

Example 6. Figure 6 shows two SCCs with more than one balanced state. Thus, these multi-battle contests are inconsistent with Centeredness. The SCC in panel (a) may be reduced to a tug-of-war, but note that the Markov property is more stringent in the reduced automaton. The SCC in panel (b) cannot be reduced to a tug-of-war.

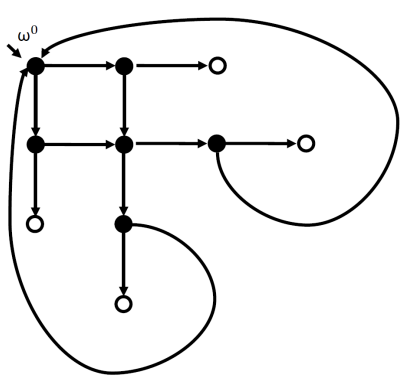

(a)

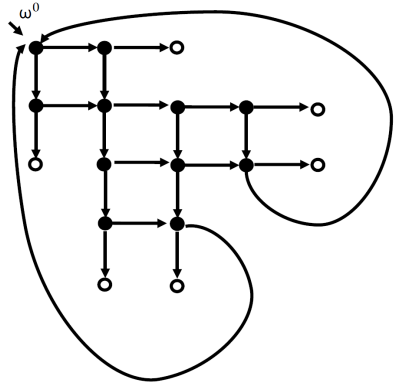

(b)

Figure 6. Two SCCs that violate Centeredness. 


\section{The tug-of-war}

The analysis above implies a central role for the tug-of-war in the analysis of multi-battle contests that can be represented by a finite state machine. In this section, we will prove that the tug-of-war admits a unique symmetric Markov perfect equilibrium. We also characterize the equilibrium. Our analysis builds upon Harris and Vickers (1987), but in contrast to their work, we will admit a different class of technologies for the component contests.

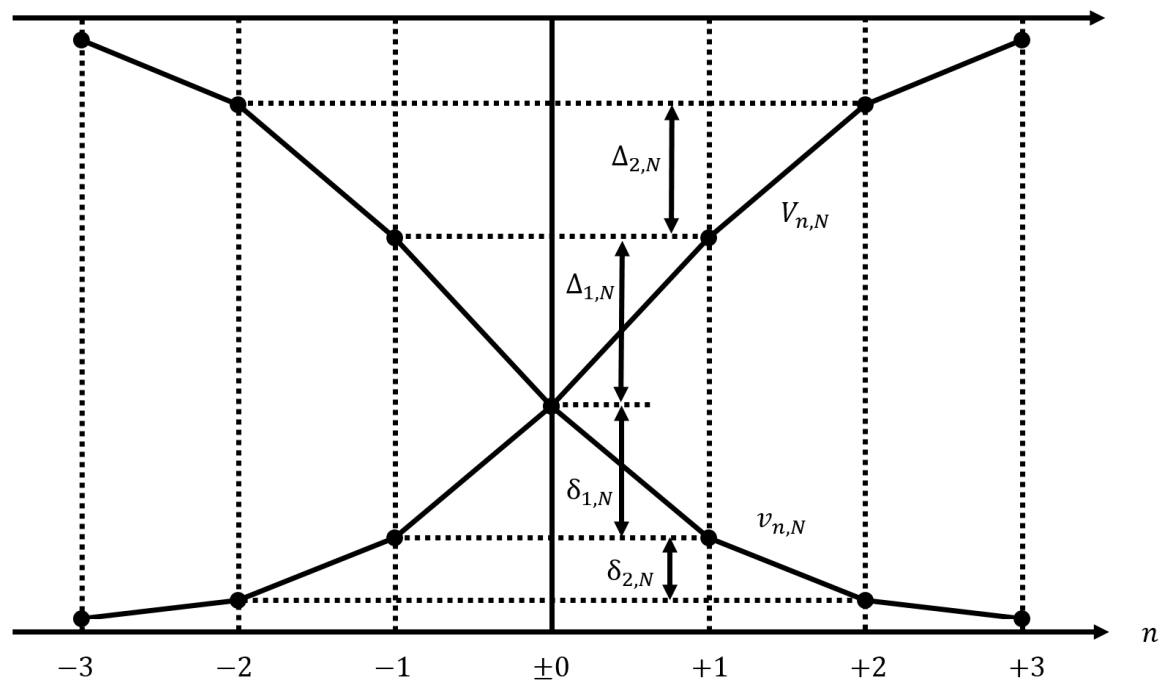

Figure 7. Continuation payoffs in the tug-of-war

Considered is a tug-of-war of distance $N \geq 1$, as introduced in Example 2. Thus, there are $2 N-1$ active states $\omega^{-(N-1)}, \ldots, \omega^{N-1}$, and two terminal states $\omega^{N} \in \Omega_{1}$ and $\omega^{-N} \in \Omega_{2}$. The initial state is $\omega^{\text {init }}=\omega^{0}$. Let $V_{n, N}$ denote the valuation, i.e., the continuation payoff, of a state $\omega_{n} \in \Omega$ for player 1 . Similarly, for player 2, the continuation payoff of state $\omega_{n} \in \Omega$ is denoted by $v_{n, N}$.

As we show in the Appendix, a Markov perfect equilibrium exists in the tug-of-war. To ensure uniqueness, however, and to characterize the equilibrium, we will impose one more assumption.

Assumption 4. $\frac{\phi(1 / \theta)}{\theta(1-\phi(\theta))}$ is strictly declining in $\theta \in(0,1)$.

The assumption captures a property of the component CSF that is crucial for constructing the Markov perfect equilibrium in the tug-of-war. Specifically, Assumption 4 ensures that, from the ratio of differences in continuation payoffs in the transition between two states state, one may uniquely determine the ratio of valuations in any of the two corresponding component battle. Assumption 4 imposes an upper 
bound on the curvature of the technology, which holds for any of the before-mentioned specifications of CSF.

Lemma 6. The Tullock, serial, and relative-difference CSF all satisfy Assumption 4.

Proof. See the Appendix.

We are now in the position to characterize the equilibrium of the tug-of-war.

Theorem 2. Suppose that Assumptions 3 and 4 hold. Let $N \geq 1$ be a positive integer. Then, the following holds true for the tug-of-war with $2 N-1$ active states:

(i) the tug-of war has a unique symmetric MPE;

(ii) $V_{n, N}$ is strictly increasing in $n \in\{-N, \ldots, N\}$, while $v_{n, N}$ is strictly declining in $n \in\{-N, \ldots, N\}$;

(iii) the symmetric equilibrium payoff $V_{0, N}=v_{0, N}$ is strictly declining in $N$;

(iv) for any fixed integer $n \geq 0$, equilibrium efforts $X_{n, N}$ for player 1 and $x_{n, N}$ for player 2 are strictly declining in $N \in\{n+1, n+2, \ldots\}$, and symmetrically, for any fixed integer $n<0$, equilibrium efforts $X_{n, N}$ for player 1 and $x_{n, N}$ for player 2 are strictly increasing in $N \in\{\ldots, n-2, n-1\}$;

(v) for $i \in\{1,2\}$, player $i$ 's probability of winning in any active state $\omega^{n}$ is independent of $N \in$ $\{n+1, n+2, \ldots\}$.

Proof. See the Appendix.

Theorem 2 establishes existence and uniqueness of the symmetric and interior Markov perfect equilibrium for a tug-of-war with an odd number of active states and constant or decreasing returns. Figure 7 illustrates equilibrium continuation payoffs at active states in a tug-of-war of distance $N=4$. These continuation values are identified recursively as the unique solution of a nonlinear equation. For $R=1$, each of these nonlinear equations is quadratic, so that an explicit solution is feasible. ${ }^{11}$

Example 7. For the case of the lottery technology (i.e., Tullock CSF with parameter $R=1$ ), and a

\footnotetext{
${ }^{11}$ The derivations are detailed in the Appendix.
} 
distance $N=2$, we get the following equilibrium continuation payoffs at active states:

$$
\begin{aligned}
& V_{0,2}=v_{0,2}=\frac{\sqrt{33}-5}{4}=0.18614 \\
& V_{1,2}=\frac{385-65 \sqrt{33}}{16}=0.72521 \\
& v_{1,2}=\frac{27 \sqrt{33}-155}{16}=6.4495 \times 10^{-3}
\end{aligned}
$$

The monotonicity of equilibrium continuation values predicted by Theorem 2(ii) is evident from these numbers.

The analysis conducted in the Appendix identifies an unexpected similarity principle across tug-of-wars with different $N$, which is that

$$
V_{n, N}-V_{n-1, N}=\frac{V_{n, N+1}-V_{n-1, N+1}}{V_{N, N+1}-V_{-N, N+1}}
$$

holds for any $n \in\{-(N-1), \ldots, N\}$. Thus, continuation payoffs known for the tug-of-war with a larger number of active states may be used to compute continuation payoffs for a tug-of-war with a smaller number of active states. For instance, scaling Example 7 down to $N=1$, relationship (10) implies for and $n=0$ that

$$
V_{0,1}=V_{0,1}-V_{-1,1}=\frac{V_{0,2}-v_{1,2}}{V_{1,2}-v_{1,2}}=0.25,
$$

which indeed corresponds to the symmetric equilibrium payoff in the one-shot lottery contest. Clearly, this property is inherited from our assumption that the contest success function is homogeneous of degree zero. As a result of the similarity property, the symmetric equilibrium payoff $V_{0, N}$ in the tugof-war is monotone also in $N$, as stated in Theorem 2(iii). For instance, the rent dissipation in the win-by-2 is always stronger than in one-shot contest, and similarly, the rent dissipation in the win-by-3 is always stronger than in a win-by-2. This is remarkable because the analogous property for the match race does not hold.

The proof of Theorem 2 that we offer in the Appendix proceeds algebraically, i.e., we solve the system of Bellman equations in a recursive way. This actually seems necessary because the abstract transformation that maps a vector of continuation payoffs, via the Bellman equations, into a new vector of continuation payoffs does not seem to possess any convenient properties. For instance, it is not a contraction. We also found that the Jacobian of this transformation is not a P-matrix, so that uniqueness is not guaranteed by the usual conditions for univalence. To see that the equilibrium strategies predicted 
by state-by-state optimization are immune against arbitrary deviations, we verify that the tug-of-war without discounting satisfies a suitable variant of the one-stage deviation principle.

\section{Existence and uniqueness}

Our second main result is the following.

Theorem 3. Impose Assumptions 1-4 and (E), (M), and (C). Then, the multi-battle contest admits a unique symmetric Markov perfect equilibrium. Moreover, the equilibrium is interior.

Proof. We start by showing that at every active state, the continuation payoff for every player at each state lies in the open interval $(0,1)$. Consider first the case where the contest has a finite horizon. Thus, there is an integer $L \geq 0$ such that the longest finite history has length $L$. Clearly, $L \geq 1$. Indeed, if $L=0$, then $\omega^{\text {init }}$ is terminal, say $\omega^{\text {init }} \in \Omega_{1}$. But then, by Assumption $2, \pi\left(\omega^{\text {init }}\right) \in \Omega_{1} \cap \Omega_{2}=\varnothing$, a contradiction. Hence, $L \geq 1$, as claimed. The proof proceeds now by induction. Suppose first that $L=1$. Then, there is precisely one active state, which is $\omega^{\text {init }}$. From Monotonicity, there is an interior equilibrium. Suppose that the claim has been shown for any finite-horizon contest of length $\leq L-1$. Take any $\omega$ such that the longest finite history in the subgame starting at $\omega$ has length $L$. There are three cases. Assume first that there is another battle at $\omega^{\prime}=\alpha(\omega, 1) \in \Omega_{\text {act }}$, but $\omega^{\prime \prime}=\alpha(\omega, 2)$ is terminal, as illustrated in panel (a) of Figure 8. Since state $\omega^{\prime \prime}$ is terminal, Monotonicity implies that player 1's payoff at $\omega^{\prime \prime}$ is zero and it suffices to show that valuation at $\omega^{\prime}$ is positive. However, given that the longest finite history in the subgame starting at $\omega^{\prime}$ has length at most $L-1$, this follows from the induction hypothesis. The situation is similar in a second case, where $\omega^{\prime}=\alpha(\omega, 1)$ is terminal, while $\omega^{\prime \prime}=\alpha(\omega, 2) \in \Omega_{\text {act }}$. See as panel (b) of Figure 8 for illustration. Here, by Monotonicity, it must be that $\omega^{\prime} \in \Omega_{1}$, and contestant 1's continuation payoff is one. Conversely, if player 1 loses and there is another battle at $\omega^{\prime \prime}=\alpha(\omega, 2)$, then by the induction hypothesis, player 1's continuation payoff is strictly below one. In a third case, neither $\omega^{\prime}$ nor $\omega^{\prime \prime}$ is terminal, as illustrated in panel (c) of Figure 8. Then, by Exchangeability, $\alpha\left(\omega^{\prime}, 2\right)=\alpha\left(\omega^{\prime \prime}, 1\right)$, and player 1's continuation payoff at $\omega^{\prime}$ must be strictly higher than player 1's continuation payoff at $\omega^{\prime \prime}$. Therefore, at $\omega$, player 1 has a positive valuation of winning. Analogous arguments can be made for player 2, of course, which proves the claim for the case of contests with finite horizon. 


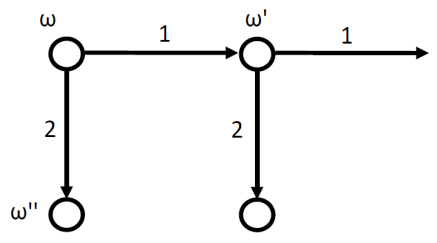

(a)

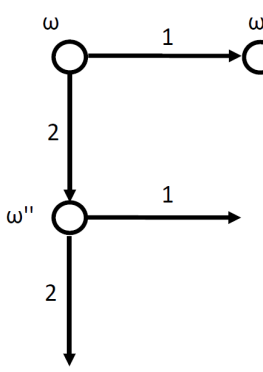

(b)

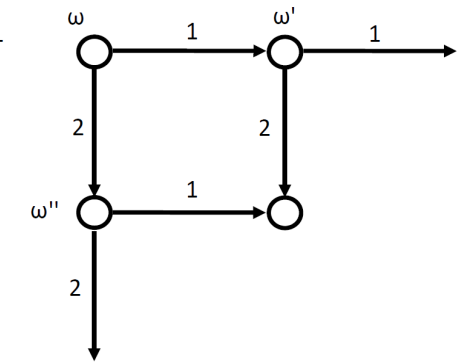

(c)

Figure 8. Proof of Theorem 3.

Consider next the case where the contest has an infinite horizon. Then, we may replace the continuation payoffs at any state in the concluding SCC by the unique and interior equilibrium payoffs of the corresponding tug-of-war of some distance $N \geq 2$. By Theorem 2 (ii), player 1's continuation payoff $V_{n, N}$ is strictly increasing, and player 2's continuation payoff $v_{n, N}$ is strictly declining in $n \in\{-N, \ldots, N\}$. The proof proceeds now precisely as above by backward induction. This proves the claim. To see that the identified profile of Markov strategies constitutes a subgame-perfect equilibrium (i.e., allowing for unilateral deviations that affect effort levels at more than one battle), one may use the same arguments as in the proof of Theorem 2 .

The following examples show that existence breaks down in general when the assumptions of Exchangeability and Monotonicity are dropped.

Example 8. Consider the multi-battle contest depicted in panel (a) of Figure 9, and suppose that $R=1$ with Tullock technology. This contest does not admit a Markov perfect equilibrium. Indeed, in the initial battle, contestants fight about whether the remaining interaction will be a one-shot contest (if 2 wins the first battle) or a win-with-2 (if 1 wins the first battle). The one-shot contest is known to yield a payoff of 0.25 for each contestant, the win-with-two a payoff of 0.18 for each contestant. Therefore, player 2 has a negative valuation of winning, so she exerts no effort. Player 1, conversely, has a positive valuation of winning, and would not want to leave the outcome to any symmetric tie-breaking rule. So player 1 has no best response. As a result, an equilibrium does not exist. While the example is asymmetric, it is simple to make it symmetric, so it is really assumption (E) that does the job here. 


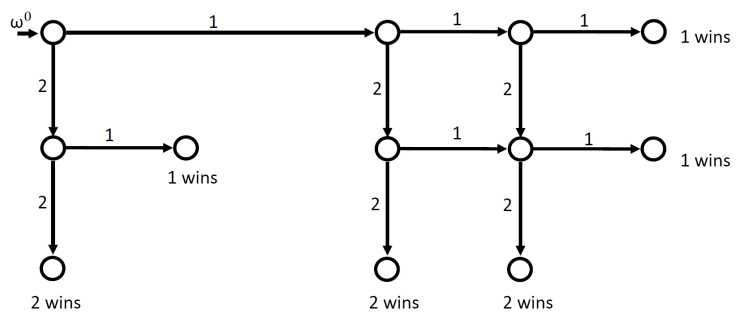

(a)

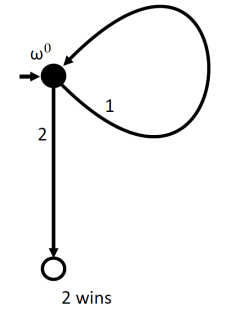

(b)

Figure 9. Multi-battle contests without MPE

Example 9. The multi-battle contest shown in panel (b) of Figure 9 is an example of nonexistence when Monotonicity does not hold, while Exchangeability holds.

Konrad and Kovenock (2009) define an important property of multi-battle contests which they refer to as pervasiveness. In short, pervasiveness means that, starting from any state corresponding to $n_{1}$ wins of player 1 and $n_{2}$ wins by player 2 , one reaches with positive probability any other state corresponding to $n_{1}^{\prime}$ wins of player 1 and $n_{2}^{\prime}$ wins by player 2 provided that $n_{1}^{\prime} \geq n_{1}$ and $n_{2}^{\prime} \geq n_{2}$. This property holds for any multi-battle contest in our class as well.

Corollary 1. Any multi-battle contest satisfying Assumptions (E), (M), and (C) is pervasive.

\section{Optimal multi-battle contests}

\subsection{Theoretical results}

In this section, we explore the intricate issue of finding the optimal dynamic structure of multi-battle contests. Of course, the degree to which the extensive form of a contest may be shaped by a third party depends on the application considered. E.g., in a sports contest, the designer has essentially full discretion (maybe up to constraints with respect to timing or players' levels of exhaustion). In contrast, a regulatory body that aims at maximizing the effort expended in R\&D activities will likely face a variety of policy constraints. All the more, the regulator's influence on the extensive form of competition will often be quite indirect. In our analysis below, however, we will abstract from such application-specific considerations.

The designer is assumed to maximize expected total efforts. Thus, we sum efforts both across players and across periods. In particular, no discounting is applied. Similar objective functions have been used in the literature (e.g., Moldovanu and Sela, 2001; Groh et al., 2006, Sec. 3.2). Note that, given that 
the prize will be allocated with probability one, we may equivalently assume that the designer aims at minimizing the players' symmetric expected equilibrium payoff in the symmetric equilibrium.

Lemma 7. Maximizing expected total efforts is equivalent to minimizing symmetric expected equilibrium payoffs in the contest.

Proof. Let $\Pi^{*}=\Pi_{1}^{*}=\Pi_{2}^{*}$ denote the symmetric equilibrium payoff. Then, $\Pi^{*}=\frac{1}{2}-e^{*}$, where $e^{*}$ denotes the total expected payoff per player. The claim is now immediate.

We can now show the following.

Theorem 4. The one-shot contest is dominated by any contest in which two consecutive battle wins at the beginning of the contest imply a win of the contest.

Proof. Take any contest in which two consecutive battle wins at the beginning of the contest imply a win of the contest. We denote by $\pi$ the continuation value of the contest after each player has won precisely one battle. Clearly, $\pi<1 / 2$. Then, the continuation payoff for a player that has lost the first battle is

$$
v \equiv \pi \phi\left(\frac{\pi}{1-\pi}\right)>0
$$

while the continuation payoff for the player having won the initial battle is

$$
V \equiv \pi+(1-\pi) \phi\left(\frac{1-\pi}{\pi}\right)<1 .
$$

Therefore, in the initial battle of the contest, the symmetric equilibrium payoff is given by

$$
\Pi^{*}=v+\sigma(V-v) .
$$

We claim that

$$
\begin{aligned}
v+\sigma(V-v) & <\sigma \\
& \Leftrightarrow \frac{v}{1-V}<\frac{\sigma}{1-\sigma} .
\end{aligned}
$$

Plugging in the expressions for $v$ and $V$, we obtain

$$
\begin{aligned}
\Pi^{*} & <\sigma \\
& \Leftrightarrow \frac{v}{1-V}<\frac{\sigma}{1-\sigma} \\
& \Leftrightarrow \frac{\pi \phi\left(\frac{\pi}{1-\pi}\right)}{(1-\pi)\left(1-\phi\left(\frac{1-\pi}{\pi}\right)\right)}<\frac{\sigma}{1-\sigma} .
\end{aligned}
$$


By Assumption 4, the left-hand side is strictly increasing in $\pi$. Moreover, for $\pi=1 / 2$, the left-hand side is identical to the right-hand side. This proves the claim, and hence, the Theorem.

The theorem shows that a very large class of dynamic contests elicits higher expected total efforts than the one-shot contest. Intuitively, this is so because even the two-fold repetition of the component contest allows making double-use of the same incentive. The conclusion of Theorem 4 can be extended by recalling that the one-shot contest is a degenerate form of the tug-of-war. Since we have shown that symmetric equilibrium payoffs in the tug-of-war are strictly declining in the number of active states, it follows that the one-shot contest is dominated, in terms of total effort, also by any non-degenerated tug-of-war. In fact, we conjecture that the one-shot contest minimizes expected total efforts across all contests. This is also suggested by the results of our numerical analysis which will be reported below.

\subsection{Numerical exploration}

In the numerical analysis, we employed several specifications. For the Tullock case, the well-known equilibrium characterization (Nti, 1999) yields

$$
\Pi_{i}^{\mathrm{TUL}}=\frac{V_{i}^{R+1}\left(V_{i}^{R}+(1-R) V_{j}^{R}\right)}{\left(V_{1}^{R}+V_{2}^{R}\right)^{2}} \quad(i, j \in\{1,2\}, j \neq i),
$$

provided that $V_{1}>0$ and $V_{2}>0$. The parameter was chosen from the grid $R \in\{0.01, \ldots, 1.00\}$. In the case of the serial specification, Alcalde and Dahm (2007) have shown that

$$
\Pi_{1}^{\mathrm{SER}}=V_{1}\left[1-\frac{1+\alpha}{2}\left(\frac{V_{2}}{V_{1}}\right)^{\alpha}\right] \text { and } \Pi_{2}^{\mathrm{SER}}=V_{2} \frac{1-\alpha}{2}\left(\frac{V_{2}}{V_{1}}\right)^{\alpha}
$$

provided that $V_{1} \geq V_{2}>0$. In this case, the parameter was chosen from the grid $\alpha \in\{0.01, \ldots, 0.99\}$, i.e., excluding the case $\alpha=1$ which implies complete rent dissipation for the contestant with the lower valuation. The relative difference CSF yields equilibrium payoffs

$$
\Pi_{i}^{\mathrm{RD}}=V_{i}\left(\frac{\tau V_{i}^{2}}{\left(V_{1}+V_{2}\right)^{2}}+\frac{1-\tau}{2}\right) \quad(i \in\{1,2\}) .
$$

In this case, the parameter was chosen from the grid $\tau \in\{0.01, \ldots, 1.00\}$.

To compare the expected equilibrium payoff across contests, we needed a way to enumerate all designs with a finite or infinite horizon. As for the finite-horizon contests, we considered multi-battle contests for which the longest sequence of consecutive battles feasible has length $(2 K+1)$, where $K \geq 1$ is an integer. One can convince oneself that the number $\mathcal{T}_{K}$ of dynamic contests with longest path $(2 K+1)$ that satisfy our assumptions corresponds to the number of nondecreasing integer sequences 
$0 \leq x_{1} \leq x_{2} \leq \ldots \leq x_{K-1}$ satisfying $x_{k} \leq k$ for any $k \in\{1, \ldots, K-1\}$. For instance, for $K=1$, there is only the best-of-three (or win-with-two) multi-battle contest. For $K=2$, there are two contests, the best-of-five (or win-with-three) contest, and the win-with-three-or-by-two contest. For larger values of $K$, the number of contests increases quickly. In fact, one can show (e.g., Pemantle and Wilf, 2009) that $\mathcal{T}_{K}$ corresponds to the $k$-th Catalan number, i.e., $\mathcal{T}_{K}=\frac{1}{K+1}\left(\begin{array}{c}2 K \\ K\end{array}\right)$, with $\mathcal{T}_{1}=1, \mathcal{T}_{2}=2, \mathcal{T}_{3}=5, \mathcal{T}_{4}=14$, and asymptotic approximation $\mathcal{T}_{K} \sim \frac{4^{K}}{K^{3 / 2} \sqrt{\pi}}$. To enumerate all possible multi-battle contests, we chose a recursive programming approach that conveniently solves the issue of an endogenous loop depth.

As for the contests with infinite horizon, these can likewise be enumerated by replacing the payoffs in the final active states of dynamic contests with finite horizon by the corresponding continuation payoffs of a tug-of-war with an odd number of active states. Figure 1(b) illustrates this idea. Note that the minimality assumption implies that the states of the concluding tug-of-war can always be arranged in the inverted L-shape. Altogether, we explored the set of (i) all finite-horizon contests that end after at most 29 battles, and (ii) all infinite-horizon contests in which a recurrent state is reached after at most 26 battles. The computations were done using Visual Basic.

\subsection{Unconstrained contests}

In our first analysis, we assume that the choice set is the entire set of multi-battle contests satisfying our assumptions. It follows from Theorem 2 that the one-shot contest is always strictly suboptimal. In fact, any tug-of-war can be strictly improved by replacing it by a longer variant. We now have the following, as we believe, interesting numerical finding.

Finding 1. The revenue maximizing multi-battle contest is always a match race of length $M \geq 2 .{ }^{12}$

To understand intuitively why the race is optimal compare the two contests shown in Figure 10. Suppose that player 1 wins the initial battle in contest (a), so that state $\omega^{(1,0)}$ is reached. Then, to win the contest, player 1 just needs one more win (at least), while player 2 would need three wins in any case. In contest (b), however, the analogous situation looks less unbalanced. Thus, the continuation value for player 1 at state $\omega^{(1,0)}$ is higher in contest (a) than in contest (b). For player 2, the situation is the opposite, i.e., the continuation value for player 2 at state $\omega^{(1,0)}$ is lower in contest (a) than in contest (b). Because of this, the net valuation of winning the initial battle is higher in contest (a) than in

\footnotetext{
${ }^{12}$ We have checked this for the Tullock, serial, and relative difference specifications, going through all finite-horizon contests that end after 12 or less consecutive battle wins by the same player. In the case of the Tullock contest, we allowed for an arbitrary concluding tie-breaking phase with up to 21 recurrent states.
} 
contest (b), whereas the continuation value for the loser of the initial battle is lower in contest (a) than in contest (b).

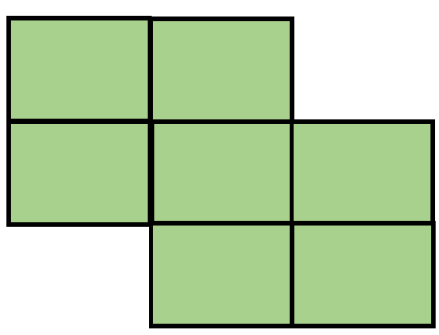

(a)

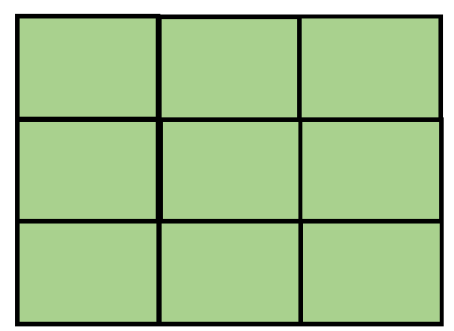

(b)

Figure 10. Optimality of the match race

It turns out that the expected payoff in the contest shown in Figure 10a, $\Pi_{w / 3 \vee b / 2}$, is always weakly higher than the expected in the best-of-five contest shown in Figure 10b, $\Pi_{w / 3}$. Analytically, this seems like a very hard problem.

For our analysis, the relative size of the changes in the continuation values across players matters. Specifically, it turns out that the change in continuation values between contests (a) and (b) is much larger for player 1 than for player 2. In other words, the leading player 1 gains, in relative terms, much more than the lagging player 2 loses. We call this the preemption effect. As a result, the equilibrium payoff, i.e., the continuation payoff at state $\omega^{\text {init }}=\omega^{(0,0)}$ is higher in contest (a) than in contest (b). In turn, this implies that contest (a) is always suboptimal from the perspective of an effort-maximizing designer. Thus, the intuitive reason for the optimality of the race is that, among all multi-battle contests, it minimizes the preemption effect by making a battle-win as incremental a progress towards winning as feasible. The one-shot multi-battle contest, however, is never optimal. The intuitive reason for this is that a repetition allows a double-use of the same prize incentives, which is impossible in a one-shot contest.

\subsection{The optimal length of a race}

Given that a match race is always optimal, the question is which length is best for a designer that maximize the expected total effort. Figure 11 illustrates that the length of the optimal race is inversely related to the Tullock parameter. Thus, intuitively, if each the contest success function reflects more randomness (i.e., a lower $R$ ), then it is preferable for the contest designer to involve contestants into a longer race. We have found the same prediction for the serial and relative difference contest. 
Finding 2. The revenue-maximizing length of a match race is monotonically increasing in the noise.

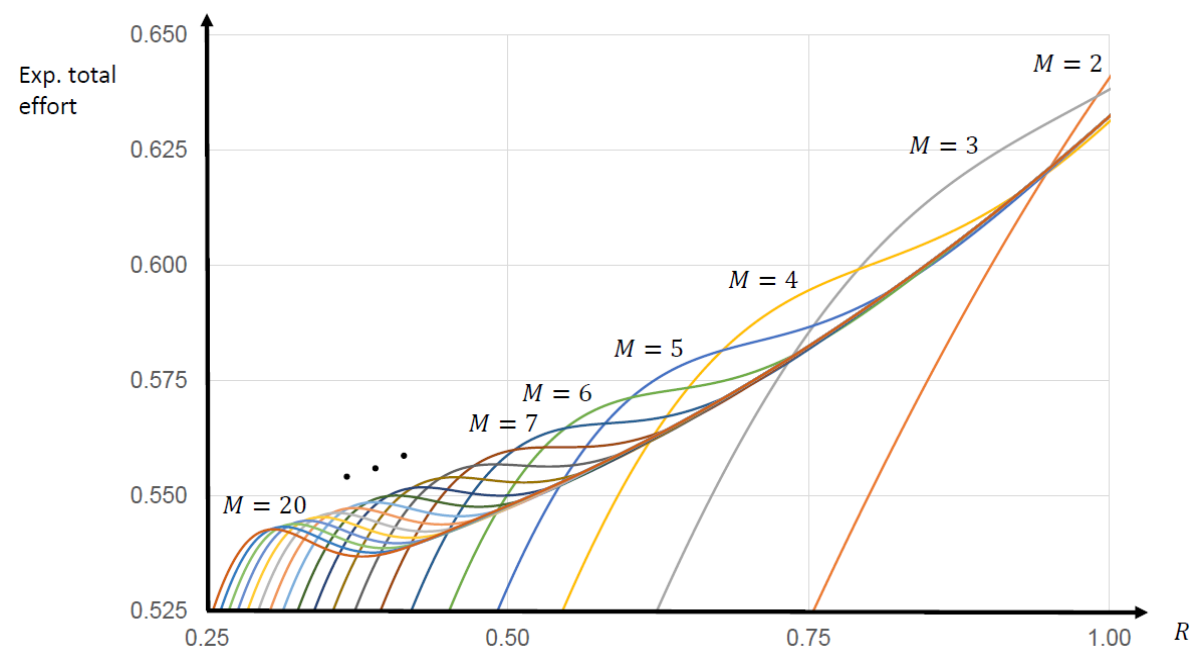

Figure 11. Expected total effort in a Tullock race, as a function of $R$

\subsection{Contests subject to a complexity bound}

In many environments, the scope for changing the rules of the contest will be more limited than assumed above. Suppose, therefore, that the contest designer is restricted to use a multi-battle contest such that the number of active states is no larger than a given number $K \geq 1$. Below, we report the results for the Tullock contest.

For $K \in\{1,2\}$, we obtain the one-shot contest. There is no other symmetric multi-battle contest under this complexity constraint.

For $K=3$, we need to compare the one-shot contest with a win-by-2. As shown in the observation, however, rent dissipation in the tug-of-war with three active states is always strictly higher than in the one-shot contest. Thus, for $K=3$, the win-by-2 is always optimal.

For $K=4$, we have the win-with-2 as an additional possibility. Numerically, the win-with-2 strictly dominates the win-by-2 if and only if $R \geq 0.89$. Intuitively, this finding captures the trade-off for the contest designer. If randomness is important ( $R$ low), then the designer has an incentive to have long multi-battle contest, so as to reward small but continued efforts provision. If, however, the constraint of the number of states is binding, then the tug-of-war, with its potentially infinite horizon, offers a better trade-off than the race.

For $K \in\{5,6\}$, the designer has the win-by-3 as an additional option. This is numerically optimal if $R<0.95$, otherwise the match race is better. This makes sense because the contest designer will make 
use of the relaxed complexity constraint.

For $K=7$, there are two additional possibilities, the win-by-4, that always dominates smaller tugof-wars. Further, we have a win-with-3-or-by-2 (Example 4). Optimal is the win-by-4 for $R<0.95$, and the win-with-2 otherwise. The win-with-3-or-by-2, however, is never optimal.

For $K=8$, the win-by-4 remains optimal for $R<0.85$, while the win-with-two (i.e., best-of-three) stays optimal for $R \geq 0.95$. In between, it is optimal to use a win-with-3-and-by-2 (Example 3 ).

For $K \in\{9,10\}$, the win-by-5 becomes optimal for $R<0.74$, while the win-with-two stays optimal for $R>0.98$. In between, it is optimal to use a win-with-three (i.e., a match race of length three).

For $K \in\{11,12\}$, the win-by- 6 replaces the win-by- 5 .

For $K=13$, the win-by-7 replaces the win-by- 6 .

For $K=14$, it is optimal to use a win-with-4-or-by-3 when $R \in[0.67, \ldots, 0.76]$.

Thus, for $R \in(0,1]$ small, the numerical always analysis finds a tug-of-war to be optimal, whereas for $R \in(0,1]$ large, the complexity constraint relaxes, and we find a match race. For intermediate values of $R$, there are cases (e.g., $K=8$ and $K=14$ ) in which we get "sporadic" contests that are optimal but neither a tug-of-war nor a match race. We have continued the analysis to state machines with up to $K=28$ active states, but this pattern remained unchanged. Recalling the relationship between complexity and the number of active states to be $K=\gamma(\mathcal{M})-2$, we may summarize our results for complexity-constrained optimal contests as follows.

Finding 3. Let the complexity $\gamma=\gamma(\mathcal{M})$ be given. Then, for any $R$ not too large, the complexity bound is binding and the optimal multi-battle contest of complexity $\gamma$ is the longest tug-of-war that can be realized with complexity $\gamma$.

\section{Conclusion}

In this paper, we have introduced three conditions that jointly define a flexible and, as we believe, natural class of symmetric multi-battle contests. This class includes both dynamic contests with finite horizon, such as the win-with- $N$ contest, also known as the best-of- $(2 N-1)$ tournament, as well as contests with infinite horizon, such as a the tug-of-war. We propose making use of a finite state machine to obtain a well-defined class of dynamic contests that can then be analyzed. It was shown that any multi-battle contest in our class has a very simple structure. Moreover, provided that the contest technology satisfies 
our assumptions, we established that any multi-battle contest admits a unique symmetric and interior Markov perfect equilibrium.

As part of the analysis, we characterized the Markov perfect equilibrium of the tug-of-war. This complements results in the existing literature. While we used the characterization result for the tug-ofwar predominantly as the basis for our numerical analysis of more general contests with infinite horizon, the result addresses also a long-standing open research question, and therefore may be of independent interest.

Finally, we have studied the optimal design of dynamic contests, where the designer sets the rules so as to maximize total aggregate effort. For this type of problem, there is still a regrettable lack of analytical methods, and so we had to resort to numerical methods. Despite this difficulty, the optimal design problem could be seen to balance a trade-off between three natural forces in multi-battle contests: (i) lower incentives due to randomness in the contest technology, (ii) a double-use of incentives, and (iii) the discouragement effect in unbalanced states. The standard race was seen to optimally balance this trade-off for a variety of contest success function if the complexity of rules is no concern for the designer. Moreover, the length of the optimal race was shown to increase monotonically in the degree of randomness in the contest technology. However, once there is a positive shadow cost of complexity, the tug-of-war is typically the optimal choice. Taken together, these findings shed light on the determinants of optimal dynamic contests.

There are some natural extensions that we chose to not address in this paper. To begin with, one could consider heterogeneous valuations of the multi-battle contest prize. That case, however, would be more demanding. For instance, we conjecture that the Markov perfect equilibrium of the tug-of-war might not be unique in that case. Next, Definition 1 could be generalized to account for, say, ties in the outcome of an individual contest, or ties in the outcome of the dynamic contest. We did not pursue this line of research because we perceive the multi-battle contest as an instrument to avoid precisely any kind of tied outcomes. Further, there might be random transitions between states. For instance, in Figure 4, an initial coin toss could determine the active state from which interaction starts. We conjecture that, incorporating such possibilities would complicate matters substantially (e.g., because it would be difficult to capture Centeredness) without yielding additional insights. Finally, one could think about having a variety of sets of final states, with different prize allocations in each of those. In that case, our assumptions would appear intuitively too tight, however. For example, in a promotional 
competition, or in a series of presidential debates, the prize allocation is usually not of a binary nature, which upholds incentives for candidates in most scenarios even if the number of battles is fixed ex-ante and independent of the outcome of individual battles.

\section{Appendix A. Analysis of the tug-of-war}

Let $N \geq 1$ be an arbitrary positive integer. We consider a tug-of-war with $2 N-1$ active states

$$
\omega^{-(N-1)}, \ldots, \omega^{-1}, \omega^{0}, \omega^{1}, \ldots, \omega^{(N-1)},
$$

and two terminal states $\omega^{N} \in \Omega_{1}$ and $\omega^{-N} \in \Omega_{2}$ (cf. Example 2). The contest success function will be kept fixed throughout. ${ }^{13}$

We start with the uniqueness part. Suppose that a Markov perfect equilibrium exists. We denote by $V_{n, N}$ and $v_{n, N}$, respectively, player 1's and player 2's continuation payoffs at state $\omega^{n}$, for any $n \in\{-N, \ldots, N\}$. In particular, $V_{N, N}=v_{-N, N}=1$ and $v_{N, N}=V_{-N, N}=0$. Moreover, we let

$$
\begin{gathered}
\Delta_{n, N}=V_{n, N}-V_{n-1, N}, \\
\delta_{n, N}=v_{n-1, N}-v_{n, N},
\end{gathered}
$$

for $n \in\{-(N-1), \ldots, N\}$, denote the change in continuation payoffs for players 1 and 2, respectively, when moving from state $\omega^{n-1}$ to $\omega^{n}$. As our first lemma shows, both $\Delta_{n, N}$ and $\delta_{n, N}$ are positive, which corresponds to the intuitive idea that winning a battle strictly raises (strictly lowers) the winner's (the loser's) continuation payoff.

Lemma A.1 In any Markov perfect equilibrium of the tug-of-war of distance $N$, we have $\Delta_{n, N}>0$ and $\delta_{n, N}>0$ for any $n \in\{-(N-1), \ldots, N\}$.

Proof. Consider any active state $\omega^{n}$, i.e., $n \in\{-(N-1), \ldots,(N-1)\}$. In the Markov perfect equilibrium, the respective effort choices $X_{n, N}$ for player 1 and $x_{n, N}$ for player 2 must form a pure-strategy Nash equilibrium in the one-shot contest with valuations $V_{n+1, N}-V_{n-1, N}$ for player 1 and $v_{n-1, N}-v_{n+1, N}$ for player 2. Moreover, by Lemma 1, for the pure-strategy Nash equilibrium to exist in the one-shot contest, we need either (i) $V_{n+1, N}-V_{n-1, N}>0$ and $v_{n-1, N}-v_{n+1, N}>0$, or (ii) $V_{n+1, N}-V_{n-1, N} \leq 0$ and

\footnotetext{
${ }^{13}$ The consideration of tug-of-wars with an even number of active states is obsolete for our analysis. We conjecture, however, that the methods developed below would allow to deal with that case as well.
} 
$v_{n-1, N}-v_{n+1, N} \leq 0$. In case (i), the equilibrium characterization of the one-shot contest (cf. Lemma 1) yields the Bellman equations

$$
\begin{aligned}
& V_{n, N}=V_{n-1, N}+\left(V_{n+1, N}-V_{n-1, N}\right) \cdot \phi\left(\frac{V_{n+1, N}-V_{n-1, N}}{v_{n-1, N}-v_{n+1, N}}\right), \\
& v_{n, N}=v_{n+1, N}+\left(v_{n-1, N}-v_{n+1, N}\right) \cdot \phi\left(\frac{v_{n-1, N}-v_{n+1, N}}{V_{n+1, N}-V_{n-1, N}}\right) .
\end{aligned}
$$

Given that equilibrium payoffs are positive in the one-shot contest, this implies that $V_{n, N}>V_{n-1, N}$ and $v_{n, N}>v_{n+1, N}$. Moreover, in this case, $X_{n, N}>0$ and $x_{n, N}>0$, so that $V_{n, N}<V_{n+1, N}$ and $v_{n, N}>v_{n+1, N}$, which proves the claim. In case (ii), $X_{n, N}=x_{n, N}=0$ by Lemma 1 , and consequently, each of the two players wins with equal probability in state $\omega^{n}$. Therefore, $V_{n+1, N} \leq V_{n, N} \leq V_{n-1, N}$ and $v_{n+1, N} \geq v_{n, N} \geq v_{n-1, N}$. By arguments detailed in case (i), this sequence of weak inequalities may be continued to both sides until $V_{N, N} \leq \ldots \leq V_{-N, N}$ and $v_{N, N} \geq \ldots \geq v_{-N, N}$, which is impossible because of $V_{N, N}=v_{-N, N}=1$ and $v_{N, N}=V_{-N, N}=0$. This concludes the analysis of case (ii), and proves the lemma.

Given Lemma A.1, we may define the ratio of changes in continuation payoffs when moving from $\omega^{n-1}$ to $\omega^{n}$,

$$
\rho_{n, N}=\frac{\delta_{n, N}}{\Delta_{n, N}}>0
$$

for any $n \in\{-(N-1), \ldots, N\}$, as well as the ratio of net valuations at any active state $\omega^{n}$,

$$
\theta_{n, N}=\frac{\delta_{n, N}+\delta_{n+1, N}}{\Delta_{n, N}+\Delta_{n+1, N}}>0
$$

i.e., for $n \in\{-(N-1), \ldots,(N-1)\}$. The following relationship exploits that the contest technology is homogeneous of degree zero, so that the ratio of net valuations at any active state uniquely determines the corresponding ratio of changes in continuation payoffs.

Lemma A.2 In any Markov perfect equilibrium of the tug-of-war with $2 N-1$ active states, continuation payoffs satisfy

$$
\theta_{n, N}=\rho_{n, N} \cdot \frac{\phi\left(1 / \theta_{n, N}\right)}{1-\phi\left(\theta_{n, N}\right)}
$$

for any $n \in\{-(N-1), \ldots,(N-1)\}$.

Proof. Fix $n \in\{-(N-1), \ldots,(N-1)\}$. Using the notation introduced above, equations (26) and (27) 
may be rewritten as

$$
\begin{aligned}
\Delta_{n, N} & =\left(\Delta_{n, N}+\Delta_{n+1, N}\right) \cdot \phi\left(\frac{\Delta_{n, N}+\Delta_{n+1, N}}{\delta_{n, N}+\delta_{n+1, N}}\right), \\
\delta_{n+1, N} & =\left(\delta_{n, N}+\delta_{n+1, N}\right) \cdot \phi\left(\frac{\delta_{n, N}+\delta_{n+1, N}}{\Delta_{n, N}+\Delta_{n+1, N}}\right) .
\end{aligned}
$$

It now follows from (32) that

$$
\begin{aligned}
\delta_{n, N} & =\left(\delta_{n, N}+\delta_{n+1, N}\right)-\delta_{n+1, N} \\
& =\left(\delta_{n, N}+\delta_{n+1, N}\right) \cdot\left\{1-\phi\left(\frac{\delta_{n, N}+\delta_{n+1, N}}{\Delta_{n, N}+\Delta_{n+1, N}}\right)\right\} .
\end{aligned}
$$

Dividing by (31), we obtain

$$
\frac{\delta_{n, N}}{\Delta_{n, N}}=\frac{\delta_{n, N}+\delta_{n+1, N}}{\Delta_{n, N}+\Delta_{n+1, N}} \cdot \frac{1-\phi\left(\frac{\delta_{n, N}+\delta_{n+1, N}}{\Delta_{n, N}+\Delta_{n+1, N}}\right)}{\phi\left(\frac{\Delta_{n, N}+\Delta_{n+1, N}}{\delta_{n, N}+\delta_{n+1, N}}\right)} .
$$

The claim is now immediate.

The following lemma shows that, for any $\rho_{n, N} \in(0,1)$, there is a unique value for $\theta_{n, N} \in(0,1)$ that solves equation (30).

Lemma A.3 For any $\rho \in(0,1)$, the mapping $\gamma_{\rho}:[0,1] \rightarrow[0,1]$ defined through

$$
\gamma_{\rho}(\theta)= \begin{cases}\rho \cdot \frac{\phi(1 / \theta)}{1-\phi(\theta)} & \text { if } \theta>0 \\ \rho & \text { if } \theta=0\end{cases}
$$

has a unique fixed point $\theta^{*} \equiv \varphi(\rho)$. Moreover, the fixed point is interior, i.e., $\varphi(\rho) \in(0,1)$.

Proof. Since

$$
\lim _{\theta \searrow 0} \phi\left(\frac{1}{\theta}\right)=\lim _{\theta \searrow 0}(1-2 f(\theta)+\phi(\theta))=1,
$$

the mapping $\gamma_{\rho}($.$) is a continuous self-mapping on the unit interval. Hence, there certainly exists a fixed$ point. This fixed point must be interior because 0 and 1 are not fixed points. Indeed, $\gamma_{\rho}(0)=\rho>0$, and

$$
\gamma_{\rho}(1)=\rho \cdot \frac{\sigma}{1-\sigma}<1,
$$

since $\sigma<\frac{1}{2}$. Finally, the fixed point $\theta^{*}$ satisfies the relationship

$$
\frac{\theta^{*}\left(1-\phi\left(\theta^{*}\right)\right)}{\phi\left(1 / \theta^{*}\right)}=\rho
$$

and hence, must be unique by Assumption 4 . 
We will need the following auxiliary result.

Lemma A.4 Assumption 3 implies $\phi(x)+\phi(1 / x)<1$, for any $x>0$.

Proof. Recall that

$$
f\left(\frac{1}{x}\right)=1-f(x)
$$

Taking the derivative of (3) yields

$$
\begin{aligned}
-\frac{1}{x^{2}} f^{\prime}\left(\frac{1}{x}\right) & =-f^{\prime}(x) \\
& \Rightarrow f^{\prime}\left(\frac{1}{x}\right)=x^{2} f^{\prime}(x) .
\end{aligned}
$$

Therefore,

$$
\begin{aligned}
\phi\left(\frac{1}{x}\right) & =f\left(\frac{1}{x}\right)-\frac{1}{x} f^{\prime}\left(\frac{1}{x}\right) \\
& =1-f(x)-x f^{\prime}(x) .
\end{aligned}
$$

From the above,

$$
\begin{aligned}
\phi(x)+\phi\left(\frac{1}{x}\right) & =f(x)-x f^{\prime}(x)+1-f(x)-x f^{\prime}(x) \\
& =1-2 x f^{\prime}(x) \\
& <1,
\end{aligned}
$$

because $f$ is strictly monotone and concave on $\mathbb{R}_{+}$.

The parameters $\theta_{n, N}$ and $\rho_{n, N}$ are now determined recursively, starting from the balanced state $\omega^{0}$.

Lemma A.5 In any symmetric Markov Perfect equilibrium of the tug-of-war with $2 N-1$ active states,

$$
\begin{aligned}
\theta_{0, N} & =1 \\
\theta_{n+1, N} & =\varphi\left(\theta_{n, N} \cdot \frac{\phi\left(\theta_{n, N}\right)}{1-\phi\left(1 / \theta_{n, N}\right)}\right) \quad(n \in\{0, \ldots, N-2\}) .
\end{aligned}
$$

Moreover, given Assumption 4, $\theta_{n+1, N}<\theta_{n, N}$ for any $n \in\{0, \ldots, N-2\}$.

Proof. Fix $N \geq 1$. Then

$$
\begin{aligned}
\theta_{0, N} & =\frac{\delta_{0, N}+\delta_{1, N}}{\Delta_{0, N}+\Delta_{1, N}} \\
& =\frac{\left(v_{-1, N}-v_{0, N}\right)+\left(v_{0, N}-v_{1, N}\right)}{\left(V_{0, N}-V_{-1, N}\right)+\left(V_{1, N}-V_{0, N}\right)} \\
& =\frac{v_{-1, N}-v_{1, N}}{V_{1, N}-V_{-1, N}} .
\end{aligned}
$$


Clearly, in any symmetric MPE, $v_{-1, N}=V_{1, N}$ and $v_{1, N}=V_{-1, N}$. Hence, $\theta_{0, N}=1$, proving (48). To prove the recursion, let $n \in\{0, \ldots, N-2\}$. Then, $n+1 \in\{1, \ldots, N-1\}$, so that by Lemma A.2, we know that

$$
\theta_{n+1, N}=\rho_{n+1, N} \cdot \frac{\phi\left(1 / \theta_{n+1, N}\right)}{1-\phi\left(\theta_{n+1, N}\right)} .
$$

Assume for the moment that $\rho_{n+1, N}<1$. Then, equation (53) says that $\theta_{n+1, N}$ is the unique fixedpoint of the mapping $\gamma_{\rho_{n+1, N}}$ defined in the statement of Lemma A.3. In this case, therefore, $\theta_{n+1, N}=$ $\varphi\left(\rho_{n+1, N}\right)$. To verify (49), it therefore suffices to show that $\rho_{n+1, N}<1$, and that

$$
\rho_{n+1, N}=\theta_{n, N} \cdot \frac{\phi\left(\theta_{n, N}\right)}{1-\phi\left(1 / \theta_{n, N}\right)}
$$

For this, we divide (31) by $\Delta_{n, N}+\Delta_{n+1, N}>0$, and get

$$
\frac{\Delta_{n, N}}{\Delta_{n, N}+\Delta_{n+1, N}}=\phi\left(1 / \theta_{n, N}\right)
$$

Taking the reciprocal on both sides and subsequently subtracting one, this becomes

$$
\frac{\Delta_{n+1, N}}{\Delta_{n, N}}=\frac{1-\phi\left(1 / \theta_{n, N}\right)}{\phi\left(1 / \theta_{n, N}\right)}
$$

Similarly, starting from (32), we get

$$
\frac{\delta_{n, N}}{\delta_{n+1, N}}=\frac{1-\phi\left(\theta_{n, N}\right)}{\phi\left(\theta_{n, N}\right)} .
$$

Multiplying (57) with (56), and rearranging, we obtain

$$
\frac{\delta_{n+1, N}}{\Delta_{n+1, N}}=\frac{\delta_{n, N}}{\Delta_{n, N}} \cdot \frac{\phi\left(\theta_{n, N}\right)}{1-\phi\left(1 / \theta_{n, N}\right)} \cdot \frac{\phi\left(1 / \theta_{n, N}\right)}{1-\phi\left(\theta_{n, N}\right)} .
$$

By Lemma A.2, we know that

$$
\frac{\phi\left(1 / \theta_{n, N}\right)}{1-\phi\left(\theta_{n, N}\right)}=\frac{\theta_{n, N}}{\rho_{n, N}} .
$$

Plugging this into equation (58), we arrive at

$$
\underbrace{\frac{\delta_{n+1, N}}{\Delta_{n+1, N}}}_{=\rho_{n+1, N}}=\underbrace{\frac{\delta_{n, N}}{\Delta_{n, N}}}_{=\rho_{n, N}} \cdot \frac{\phi\left(\theta_{n, N}\right)}{1-\phi\left(1 / \theta_{n, N}\right)} \cdot \frac{\theta_{n, N}}{\rho_{n, N}},
$$

which yields (54). Finally, Lemma A.4 guarantees that

$$
\phi\left(\theta_{n, N}\right)+\phi\left(1 / \theta_{n, N}\right)<1 .
$$


Hence, $\theta_{n, N} \leq 1$ and equation (54) imply that $\rho_{n+1, N}<1$. This proves the the first part of the lemma. To prove the second part, let $n \in\{0, \ldots, N-2\}$. Then, (49) implies that

$$
\theta_{n+1, N}=\theta_{n, N} \cdot \frac{\phi\left(\theta_{n, N}\right)}{1-\phi\left(1 / \theta_{n, N}\right)} \frac{\phi\left(1 / \theta_{n+1, N}\right)}{1-\phi\left(\theta_{n+1, N}\right)} .
$$

Re-writing yields

$$
\frac{\theta_{n, N} \phi\left(\theta_{n, N}\right)}{1-\phi\left(1 / \theta_{n, N}\right)}=\frac{\theta_{n+1, N}\left(1-\phi\left(\theta_{n+1, N}\right)\right)}{\phi\left(1 / \theta_{n+1, N}\right)} .
$$

To provoke a contradiction, suppose that $\theta_{n+1, N} \geq \theta_{n, N}$. Then, by Assumption 4,

$$
\frac{\theta_{n+1, N}\left(1-\phi\left(\theta_{n+1, N}\right)\right)}{\phi\left(1 / \theta_{n+1, N}\right)} \geq \frac{\theta_{n, N}\left(1-\phi\left(\theta_{n, N}\right)\right)}{\phi\left(1 / \theta_{n, N}\right)} .
$$

Combining the last two inequality delivers

$$
\frac{\theta_{n, N} \phi\left(\theta_{n, N}\right)}{1-\phi\left(1 / \theta_{n, N}\right)} \geq \frac{\theta_{n, N}\left(1-\phi\left(\theta_{n, N}\right)\right)}{\phi\left(1 / \theta_{n, N}\right)}
$$

which reduces to

$$
1 \leq \phi\left(\theta_{n, N}\right)+\phi\left(1 / \theta_{n, N}\right)
$$

But this is in conflict with Lemma A.4. The contradiction proves the second part of the lemma, which concludes the proof.

Next, we consider ratios

$$
\begin{aligned}
\widehat{\Delta}_{n, N}=\frac{\Delta_{n, N}}{\Delta_{0, N}} & (n \in\{0,1, \ldots, N\}), \\
\widehat{\delta}_{n, N}=\frac{\delta_{n, N}}{\delta_{1, N}} & (n \in\{1, \ldots, N\}) .
\end{aligned}
$$

where, in any symmetric Markov perfect equilibrium, $\Delta_{0, N}=V_{0, N}-V_{-1, N}=v_{0, N}-v_{1, N}=\delta_{1, N}$. The following lemma allows to recursively compute $\widehat{\Delta}_{n, N}$ and $\widehat{\delta}_{n, N}$ from the previously determined parameters $\theta_{n, N}$.

Lemma A.6 We have $\widehat{\Delta}_{0, N}=\widehat{\delta}_{1, N}=1$, and

$$
\begin{aligned}
\widehat{\Delta}_{n+1, N}=\widehat{\Delta}_{n, N} \cdot \frac{1-\phi\left(1 / \theta_{n+1, N}\right)}{\phi\left(1 / \theta_{n+1, N}\right)} & (n \in\{0,1, \ldots, N-1\}), \\
\widehat{\delta}_{n+1, N}=\widehat{\delta}_{n, N} \cdot \frac{\phi\left(\theta_{n+1, N}\right)}{1-\phi\left(\theta_{n+1, N}\right)} & (n \in\{1, \ldots, N-1\}) .
\end{aligned}
$$

Proof. By definition, $\widehat{\Delta}_{0, N}=\widehat{\delta}_{1, N}=1$. Further, the recursive relationships (69) and (70) follow immediately from equations (56) and (57), respectively. 
Lemma A.7 Let $N \geq 1$. Then, continuation payoffs in any symmetric Markov perfect equilibrium satisfy

$$
\begin{aligned}
V_{n, N}=\frac{\sum_{\nu=1}^{N} \widehat{\delta}_{\nu, N}+\sum_{\nu=1}^{n} \widehat{\Delta}_{\nu, N}}{\sum_{\nu=1}^{N} \widehat{\delta}_{\nu, N}+\sum_{\nu=1}^{N} \widehat{\Delta}_{\nu, N}} & (n=0, \ldots, N) \\
v_{n, N}=\frac{\sum_{\nu=n+1}^{N} \widehat{\delta}_{\nu, N}}{\sum_{\nu=1}^{N} \widehat{\delta}_{\nu, N}+\sum_{\nu=1}^{N} \widehat{\Delta}_{\nu, N}} & (n=0, \ldots, N) .
\end{aligned}
$$

Proof. We start by rewriting the sums appearing in equations (71) and (72). This yields for any $n \in\{0, \ldots, N+1\}$

$$
\begin{aligned}
\sum_{\nu=1}^{n} \widehat{\Delta}_{\nu, N} & =\frac{1}{\Delta_{0, N}} \sum_{\nu=1}^{n} \Delta_{\nu, N} \\
& =\frac{1}{\Delta_{0, N}} \sum_{\nu=1}^{n}\left(V_{\nu, N}-V_{\nu-1, N}\right) \\
& =\frac{V_{n, N}-V_{0, N}}{\Delta_{0, N}} \\
& =\frac{V_{n, N}-V_{0, N}}{\delta_{1, N}} .
\end{aligned}
$$

In particular, for $n=N$, we obtain

$$
\sum_{\nu=1}^{N} \widehat{\Delta}_{\nu, N}=\frac{1-V_{0, N}}{\delta_{1, N}}
$$

Similarly,

$$
\begin{aligned}
\sum_{\nu=n+1}^{N} \widehat{\delta}_{\nu, N} & =\frac{1}{\delta_{1, N}} \sum_{\nu=n+1}^{N} \delta_{\nu, N} \\
& =\frac{1}{\delta_{1, N}} \sum_{\nu=n+1}^{N}\left(v_{\nu-1, N}-v_{\nu, N}\right) \\
& =\frac{v_{n, N}-v_{N, N}}{\delta_{1, N}} \\
& =\frac{v_{n, N}}{\delta_{1, N}}
\end{aligned}
$$

In particular, for $n=0$,

$$
\sum_{\nu=1}^{N} \widehat{\delta}_{\nu, N}=\frac{v_{0, N}}{\delta_{1, N}}
$$

Plugging this all in the right-hand side of (71), and subsequently eliminating the common denominator $\delta_{1, N}$, delivers

$$
\frac{\sum_{\nu=1}^{N} \widehat{\delta}_{\nu, N}+\sum_{\nu=1}^{n} \widehat{\Delta}_{\nu, N}}{\sum_{\nu=1}^{N} \widehat{\delta}_{\nu, N}+\sum_{\nu=1}^{N} \widehat{\Delta}_{\nu, N}}=\frac{v_{0, N}+V_{n, N}-V_{0, N}}{v_{0, N}+1-V_{0, N}}=V_{n, N}
$$


as claimed. In an analogous fashion, we obtain

$$
\frac{\sum_{\nu=n+1}^{N} \widehat{\delta}_{\nu, N}}{\sum_{\nu=1}^{N} \widehat{\delta}_{\nu, N}+\sum_{\nu=1}^{N} \widehat{\Delta}_{\nu, N}}=\frac{v_{n, N}}{v_{0, N}+1-V_{0, N}}=v_{n, N} .
$$

This proves the lemma.

Proof of Theorem 2. (i) (Uniqueness) Note that Lemma A.7 above settles uniqueness of the symmetric Markov perfect equilibrium. (Existence) From the continuation payoffs identified above, a candidate equilibrium may be constructed in a straightforward way. Indeed, by the virtue of Lemma 1, it suffices to set equilibrium efforts for player 1 and 2 , respectively, at any active state $\omega^{n}$ equal to

$$
\begin{aligned}
& X_{n, N}^{*}=\left(v_{n-1, N}-v_{n+1, N}\right) f^{\prime}\left(\frac{v_{n-1, N}-v_{n+1, N}}{V_{n+1, N}-V_{n-1, N}}\right), \\
& x_{n, N}^{*}=\left(V_{n+1, N}-V_{n-1, N}\right) f^{\prime}\left(\frac{V_{n+1, N}-V_{n-1, N}}{v_{n-1, N}-v_{n+1, N}}\right),
\end{aligned}
$$

where $n \in\{-(N-1), \ldots,(N-1)\}$. Clearly, this defines a symmetric and interior strategy profile with the property that unilateral deviations at a given finite history are not profitable. It remains to be shown that, in the spirit of the one-stage deviation principle, simultaneous deviations at any set of histories are not profitable. For this, note that the opponent's strategy is fixed and interior. Moreover, since a deviating player never exerts an effort in a battle in excess of the value of the prize for the entire contest, it may be assumed that efforts in each battle are taken from the interval $[0,1]$. Suppose that a pure strategy exists that grants a player an expected payoff of at least $\varepsilon>0$ higher than the expected payoff in the candidate equilibrium. Then, there is a time $T$ such that, for any given length of play exceeding $T$, the deviator's probability for winning the contest after $T$ stages is strictly smaller than $\varepsilon$. Therefore, there exists a profitable deviation with finite horizon as well, and hence, a profitable deviation at a single state. Thus, we have established existence as well, which concludes the proof of part (i) of Theorem 2.

(ii) Recall that $V_{-n, N}=v_{n, N}$ and $v_{-n, N}=V_{-n, N}$, for any $n \in\{0, \ldots, N\}$. Therefore, it clearly suffices to prove, in view of Lemma A.7, that $\widehat{\delta}_{n, N}>0$ for any $n \in\{1, \ldots, N\}$, and that $\widehat{\Delta}_{n, N}>0$ for any $n \in\{1, \ldots, N\}$ for any $n \in\{1, \ldots, N\}$. Now, a straightforward induction argument involving Lemmas A.3 and A.5 shows that $\theta_{n, N}>0$ for any $n \in\{0, \ldots, N-1\}$. Therefore, via Lemma A.6, it follows that, indeed, $\widehat{\delta}_{n, N}>0$ for any $n \in\{1, \ldots, N\}$, and $\widehat{\Delta}_{n, N}>0$ for any $n \in\{1, \ldots, N\}$ for any $n \in\{1, \ldots, N\}$. This delivers part (ii). 
(iii) As illustrated in Figure 7, when moving from state $\omega^{n-1}$ to $\omega^{n}$, for any $n \in\{1, \ldots, N\}$, player 1 's increase in the continuation payoff when moving one state to the right is always larger in absolute value than player 2's corresponding loss in the continuation payoff. Formally,

$$
\frac{\delta_{n, N}}{\Delta_{n, N}}=\rho_{n, N}<1
$$

for any $n \in\{1, \ldots, N\}$, as has been checked in the proof of Lemma A.5. Therefore, the symmetric equilibrium payoff indeed declines strictly in $N$. This proves part (iii).

(iv) From equations (85-86) and the similarity principle, player 1's and player 2's respective equilibrium efforts $X_{n, N}$ and $x_{n, N}$ in each active state $\omega^{n}$ are strictly declining in $N$, i.e., $X_{n, N+1}<X_{n, N}$ and $x_{n, N+1}<x_{n, N}$ for any $n \in\{-(N-1), \ldots, N-1\}$. This proves (iv).

(v) As the ratio of efforts does not change, i.e., $x_{n, N+1} / X_{n, N+1}=x_{n, N} / X_{n, N}$ for any $n \in\{-(N-$ 1), .., $N-1$ \}, winning probabilities at active states do not change in the transition from $N$ to $N+1$. This proves part (v), and hence, the theorem.

The next lemma is a formal statement of the similarity principle mentioned in the body of the paper.

Lemma A.8 (Similarity property) The parameters $\theta_{n, N}, \widehat{\Delta}_{n, N}$, and $\widehat{\delta}_{n, N}$ do not depend on $N$.

Proof. By Lemma A.5, have $\theta_{0, N}=1$ for any $N \geq 1$. Since the recursion does not depend on $N$, the parameter $\theta_{n, N}$ does not depend on $N$ either. The other claims are now immediate from Lemma A.6.

Details on Example 7. In the case of the Tullock CSF with parameter $R=1$, the fixed point identified by Lemma A.3 admits the explicit representation

$$
\varphi_{1}(\rho)=\frac{1}{4}(\sqrt{1+8 \rho}-1) .
$$

Hence, $\theta_{0}=1$, and

$$
\theta_{1}=\varphi\left(\frac{\theta_{0}^{2}}{2+\theta_{0}}\right)=\varphi\left(\frac{1}{3}\right)=\frac{\sqrt{33}}{12}-\frac{1}{4}=0.22871 .
$$

Moreover, $\widehat{\Delta}_{0}=\widehat{\delta}_{1}=1$, and

$$
\begin{aligned}
& \widehat{\Delta}_{1}=\widehat{\Delta}_{0} \cdot \theta_{0} \cdot\left(2+\theta_{0}\right)=3, \\
& \widehat{\Delta}_{2}=\widehat{\Delta}_{1} \cdot \theta_{1} \cdot\left(2+\theta_{1}\right)=\frac{3 \sqrt{33}-5}{8}=1.5292, \\
& \widehat{\delta}_{2}=\widehat{\delta}_{1} \cdot \frac{\theta_{1}^{2}}{1+2 \theta_{1}}=\frac{5 \sqrt{33}-27}{48}=3.5892 \times 10^{-2} .
\end{aligned}
$$


Finally, from Lemma A.7,

$$
\begin{aligned}
& V_{0,2}=v_{0,2}=\frac{\widehat{\delta}_{1}+\widehat{\delta}_{2}}{\widehat{\delta}_{1}+\widehat{\delta}_{2}+\widehat{\Delta}_{1}+\widehat{\Delta}_{2}}=\frac{\sqrt{33}-5}{4}=0.18614, \\
& V_{1,2}=\frac{\widehat{\delta}_{1}+\widehat{\delta}_{2}+\widehat{\Delta}_{1}}{\widehat{\delta}_{1}+\widehat{\delta}_{2}+\widehat{\Delta}_{1}+\widehat{\Delta}_{2}}=\frac{385-65 \sqrt{33}}{16}=0.72521, \\
& v_{1,2}=\frac{\widehat{\delta}_{2}}{\widehat{\delta}_{1}+\widehat{\delta}_{2}+\widehat{\Delta}_{1}+\widehat{\Delta}_{2}}=\frac{27 \sqrt{33}-155}{16}=6.4495 \times 10^{-3},
\end{aligned}
$$

which concludes the derivation of the continuation payoffs in the case $N=2$.

\section{Appendix B. Proofs of Lemmas 1 and 6}

Proof of Lemma 1. If $p$ is constant, then anonymity implies $p \equiv 0.5$, so that $x_{1}^{*}=x_{2}^{*}=0$. Moreover, $\Pi_{1}^{*}=V_{1} / 2>0$ and $\Pi_{2}^{*}=V_{2} / 2>0$, which proves the claim in this case. Assume, therefore, that $p$ is not constant. For that case, Malueg and Yates (2005, Lemma 1) have shown that any pure-strategy equilibrium satisfies $x_{1}^{*}>0$ and $x_{2}^{*}>0$. For $\xi>0$, we may define $f(\xi)=p(\xi, 1)$. Then we have by homogeneity that

$$
\begin{aligned}
& \Pi_{1}\left(x_{1}, x_{2}\right)=f\left(\frac{x_{1}}{x_{2}}\right) V_{1}-x_{1} \\
& \Pi_{2}\left(x_{1}, x_{2}\right)=f\left(\frac{x_{2}}{x_{1}}\right) V_{2}-x_{2}
\end{aligned}
$$

for any $x_{1}>0$ and $x_{2}>0$. The necessary first-order conditions for an interior optimum read

$$
\begin{aligned}
& f^{\prime}\left(\frac{x_{1}^{*}}{x_{2}^{*}}\right) \frac{V_{1}}{x_{2}^{*}}-1=0 \\
& f^{\prime}\left(\frac{x_{2}^{*}}{x_{1}^{*}}\right) \frac{V_{2}}{x_{1}^{*}}-1=0
\end{aligned}
$$

Combining anonymity with homogeneity, we get

$$
f\left(\frac{x_{1}}{x_{2}}\right)+f\left(\frac{x_{2}}{x_{1}}\right)=1
$$

for any $x_{1}>0$ and $x_{2}>0$. Differentiating w.r.t. $x_{1}$ yields

$$
\frac{1}{x_{2}} f^{\prime}\left(\frac{x_{1}}{x_{2}}\right)-\frac{x_{2}}{x_{1}^{2}} f^{\prime}\left(\frac{x_{2}}{x_{1}}\right)=0
$$

so that (a variant of Euler's Lemma)

$$
f^{\prime}\left(\frac{x_{2}}{x_{1}}\right)=f^{\prime}\left(\frac{x_{1}}{x_{2}}\right)\left(\frac{x_{1}}{x_{2}}\right)^{2},
$$


for any $x_{1}>0$ and $x_{2}>0$. Evaluating at $x_{1}=x_{1}^{*}$ and $x_{2}=x_{2}^{*}$, and combining the resulting relationship with (98) and (99), we arrive at

$$
\frac{x_{1}^{*}}{x_{2}^{*}}=\frac{V_{1}}{V_{2}}
$$

as in Baik (2004) and Malueg and Yates (2005). Moreover, from (98),

$$
\begin{aligned}
x_{1}^{*} & =V_{2} f^{\prime}\left(\frac{V_{2}}{V_{1}}\right) \\
& =V_{2} f^{\prime}\left(\frac{V_{1}}{V_{2}}\right)\left(\frac{V_{1}}{V_{2}}\right)^{2} \\
& =V_{1}^{2} \frac{\partial p\left(V_{1}, V_{2}\right)}{\partial x_{1}} .
\end{aligned}
$$

Analogously, for player 2,

$$
x_{2}^{*}=-V_{2}^{2} \frac{\partial p\left(V_{1}, V_{2}\right)}{\partial x_{2}}=V_{2}^{2} \frac{\partial p\left(V_{2}, V_{1}\right)}{\partial x_{1}},
$$

as claimed. Thus, we have shown that, provided that a pure-strategy Nash equilibrium exists, it is unique and given by efforts (106) and (107). To see that these effort levels constitute a pure-strategy Nash equilibrium, note that the equilibrium payoff function for player 1 ,

$$
\widetilde{\Pi}_{1}\left(x_{1}\right)=p\left(x_{1}, x_{2}^{*}\right) V_{1}-x_{1}
$$

is concave on $\mathbb{R}_{+}$. Hence, given that the first-order condition holds, $x_{1}^{*}$ is indeed a global maximum of $\widetilde{\Pi}_{1}$. Finally, it is obvious that equilibrium profits are positive if the CSF is noncompetitive. To see that equilibrium payoffs (5) are positive if the CSF is strictly concave, recall that a player can always ensure a nonnegative payoff by choosing an effort of zero. Since $x_{1}^{*}>0$ is the unique interior maximum, strict concavity implies that $\Pi_{1}^{*}>0$. A similar argument shows that $\Pi_{2}^{*}>0$.

Proof of Lemma 6. In the Tullock case, $\phi^{\mathrm{TUL}}(\xi)=\frac{\xi^{R}\left(\xi^{R}+1-R\right)}{\left(1+\xi^{R}\right)^{2}}$, with $R \in(0,1]$, so that

$$
\begin{aligned}
\frac{\phi^{\mathrm{TUL}}(1 / \theta)}{\phi^{\mathrm{TUL}}(1-\phi(\theta))} & =\frac{\theta^{-R}\left(\theta^{-R}+1-R\right)}{\theta\left(1+\theta^{-R}\right)^{2}\left(1-\frac{\theta^{R}\left(\theta^{R}+1-R\right)}{\left(1+\theta^{R}\right)^{2}}\right)} \\
& =\frac{\theta^{R}\left(\theta^{-R}+1-R\right)}{\theta\left(1+\theta^{R}\right)^{2}\left(1-\frac{\theta^{R}\left(\theta^{R}+1-R\right)}{\left(1+\theta^{R}\right)^{2}}\right)} \\
& =\frac{\theta^{R}\left(\theta^{-R}+1-R\right)}{\theta\left(1+\theta^{R}\right)^{2}-\theta^{R}\left(\theta^{R}+1-R\right)} \\
& =\frac{1}{\theta} \frac{\theta^{R}(1-R)+1}{\theta^{R}(1+R)+1},
\end{aligned}
$$

which is indeed strictly declining. 
In the case of the serial contest with parameter $\alpha \in(0,1)$, we have $\phi^{\mathrm{SER}}(x)=1-\frac{1+\alpha}{2} x^{-\alpha}$ if $x \geq 1$, and $\phi^{\operatorname{SER}}(x)=\frac{1-\alpha}{2} x^{\alpha}$ if $x<1$. Note that this is a continuous function (not differentiable though). To check if Assumption 4 holds, we note that for $x \geq 1$,

$$
\frac{\phi^{\mathrm{SER}}(1 / x)}{x\left(1-\phi^{\mathrm{SER}}(x)\right)}=\frac{\frac{1-\alpha}{2} x^{-\alpha}}{x\left(\frac{1+\alpha}{2} x^{-\alpha}\right)}=\frac{1-\alpha}{x(1+\alpha)},
$$

which is strictly declining in $x$, and for $x<1$,

$$
\frac{\phi^{\mathrm{SER}}(1 / x)}{x\left(1-\phi^{\mathrm{SER}}(x)\right)}=\frac{1-\frac{1+\alpha}{2} x^{\alpha}}{x\left(1-\frac{1-\alpha}{2} x^{\alpha}\right)},
$$

with derivative

$$
\frac{\partial}{\partial x} \frac{\phi^{\mathrm{SER}}(1 / x)}{x\left(1-\phi^{\mathrm{SER}}(x)\right)}=(-4) \cdot \frac{\left(2-x^{\alpha}\right)^{2}+\left(4-x^{\alpha}\right) \alpha^{2} x^{\alpha}}{x^{2}\left(1-\frac{1-\alpha}{2} x^{\alpha}\right)^{2}}<0 .
$$

Thus, Assumption 4 holds also for the serial contest.

In the case of the relative difference contest with parameter $\tau \in(0,1]$, we have

$$
\phi^{\mathrm{RD}}(x)=\frac{\tau x^{2}}{(x+1)^{2}}+\frac{1-\tau}{2} .
$$

To check that Assumption 4 holds, we note that

$$
\begin{aligned}
\frac{\phi^{\mathrm{RD}}(1 / x)}{x\left(1-\phi^{\mathrm{RD}}(x)\right)} & =\frac{\frac{\tau}{(x+1)^{2}}+\frac{1-\tau}{2}}{x\left(1-\frac{\tau x^{2}}{(x+1)^{2}}-\frac{1-\tau}{2}\right)} \\
& =\frac{\tau+\frac{1-\tau}{2}(x+1)^{2}}{x\left(\left(1-\frac{1-\tau}{2}\right)(x+1)^{2}-\tau x^{2}\right)} \\
& =\frac{(1-\tau) x^{2}+2(1-\tau) x+1+\tau}{x\left((1-\tau) x^{2}+2(1+\tau) x+1+\tau\right)},
\end{aligned}
$$

Brute-force derivation shows that

$$
\begin{aligned}
& \frac{\partial}{\partial x} \frac{\phi^{\mathrm{RD}}(1 / x)}{x\left(1-\phi^{\mathrm{RD}}(x)\right)} \\
& =-\frac{x\left(x^{3}+4 x^{2}+4\right)(1-\tau)^{2}+6 x^{2}\left(1-\tau^{2}\right)+16 x \tau+(1+\tau)^{2}}{x^{2}\left((1-\tau) x^{2}+2(1+\tau) x+1+\tau\right)^{2}} \\
& <0
\end{aligned}
$$

Thus, Assumption 4 holds also for the relative difference contest.

\section{Appendix C. Details on the contest specifications used in the paper}

Tullock. If $p\left(x_{1}, x_{2}\right)=p_{1}^{\mathrm{TUL}}\left(x_{1}, x_{2}\right)$, then the unique pure-strategy Nash equilibrium is given by effort levels

$$
x_{1}^{\mathrm{TUL}}=\frac{R V_{1}^{R+1} V_{2}^{R}}{\left(V_{1}^{R}+V_{2}^{R}\right)^{2}} \text { and } x_{2}^{\mathrm{TUL}}=\frac{R V_{1}^{R} V_{2}^{R+1}}{\left(V_{1}^{R}+V_{2}^{R}\right)^{2}} .
$$


Moreover, equilibrium payoffs are given as

$$
\Pi_{1}^{\mathrm{TUL}}=\frac{V_{1}^{R+1}\left(V_{1}^{R}+(1-R) V_{2}^{R}\right)}{\left(V_{1}^{R}+V_{2}^{R}\right)^{2}} \text { and } \Pi_{2}^{\mathrm{TUL}}=\frac{V_{2}^{R+1}\left(V_{2}^{R}+(1-R) V_{1}^{R}\right)}{\left(V_{1}^{R}+V_{2}^{R}\right)^{2}} .
$$

Serial. If $p\left(x_{1}, x_{2}\right)=p_{1}^{\mathrm{SER}}\left(x_{1}, x_{2}\right)$, then the unique pure-strategy Nash equilibrium, if $V_{1} \geq V_{2}$, is given by efforts

$$
x_{1}^{\mathrm{SER}}=\frac{\alpha V_{1}}{2}\left(\frac{V_{2}}{V_{1}}\right)^{\alpha} \text { and } x_{2}^{\mathrm{SER}}=\frac{\alpha V_{2}}{2}\left(\frac{V_{2}}{V_{1}}\right)^{\alpha} .
$$

Moreover, payoffs are given as

$$
\Pi_{1}^{\mathrm{SER}}=V_{1}\left[1-\frac{1+\alpha}{2}\left(\frac{V_{2}}{V_{1}}\right)^{\alpha}\right] \text { and } \Pi_{2}^{\mathrm{SER}}=V_{2} \frac{(1-\alpha)}{2}\left(\frac{V_{2}}{V_{1}}\right)^{\alpha}
$$

Cf. Alcalde and Dahm (2007, Th. 3.4).

Relative-difference CSF. We have

$$
\begin{aligned}
f^{\mathrm{RD}}(x) & =\frac{\tau x}{x+1}+\frac{1-\tau}{2}, \\
\phi^{\mathrm{RD}}(x) & =\frac{\tau x}{x+1}+\frac{1-\tau}{2}-\frac{\tau x}{(x+1)^{2}} \\
& =\frac{\tau x^{2}}{(x+1)^{2}}+\frac{1-\tau}{2} .
\end{aligned}
$$

This yields equilibrium efforts

$$
x_{1}^{\mathrm{RD}}=\frac{\tau V_{1}^{2} V_{2}}{\left(V_{1}+V_{2}\right)^{2}} \text { and } x_{2}^{\mathrm{RD}}=\frac{\tau V_{1} V_{2}^{2}}{\left(V_{1}+V_{2}\right)^{2}},
$$

and

$$
\Pi_{1}^{\mathrm{RD}}=V_{1}\left(\frac{\tau V_{1}^{2}}{\left(V_{1}+V_{2}\right)^{2}}+\frac{1-\tau}{2}\right) \text { and } \Pi_{2}^{\mathrm{RD}}=V_{2}\left(\frac{\tau V_{2}^{2}}{\left(V_{1}+V_{2}\right)^{2}}+\frac{1-\tau}{2}\right)
$$

\section{References}

Agastya, M., McAfee, R.P. (2006), Continuing wars of attrition, available at: SSRN 935970.

Alcalde, J., Dahm, M. (2007), Tullock and Hirshleifer: A meeting of the minds, Review of Economic Design 11, 101-124.

Baik, K.H. (2004), Two-player asymmetric contests with ratio-form contest success functions, Economic Inquiry 42, 679-689. 
Beviá, C., Corchón, L.C. (2015), Relative difference contest success function, Theory and Decision 78, $377-398$

Blavatskyy, P.R. (2010), Contest success function with the possibility of a draw: Axiomatization, Journal of Mathematical Economics 46, 267-276.

Budd, C., Harris, C., Vickers, J. (1993), A model of the evolution of duopoly-does the asymmetry between firms tend to increase or decrease?, Review of Economic Studies 60, 543-573.

Dechenaux, E., Kovenock, D., Sheremeta, R.M. (2015), A survey of experimental research on contests, all-pay auctions and tournaments, Experimental Economics 18, 609-669.

Deck, C.A., Sheremeta, R.M. (2015), Tug-of-war in the laboratory, Chapman University.

Dixit, A. (1987), Strategic behavior in contests, American Economic Review 77, 891-898.

Doğan, S., Karagözoğlu, E., Keskin, K., Sağlam, Ç. (2018), Multi-player race, Journal of Economic Behavior \& Organization 149, 123-136.

Doraszelski, U., Markovich, S. (2007), Advertising dynamics and competitive advantage, RAND Journal of Economics 38, 557-592.

Edwards, P.M. (2000), To Acknowledge a War: The Korean War in American Memory (No. 193), Greenwood Publishing Group.

Ericson, R., Pakes, A. (1995), Markov-perfect industry dynamics: A framework for empirical work, Review of Economic Studies 62, 53-82.

Fu, Q., Lu, J., Pan, Y. (2015), Team contests with multiple pairwise battles, American Economic Review 105, 2120-2140.

Fudenberg, D., Gilbert, R., Stiglitz, J., Tirole, J. (1983), Preemption, leapfrogging and competition in patent races, European Economic Review 22, 3-31.

Groh, C., Moldovanu, B., Sela, A., Sunde, U. (2012), Optimal seedings in elimination tournaments, Economic Theory 49, 59-80.

Grossman, G.M., Shapiro, C. (1987), Dynamic R\&D competition, Economic Journal 97, 372-387.

Häfner, S. (2017), A tug-of-war team contest, Games and Economic Behavior 104, 372-391.

Harris, C., Vickers, J. (1987), Racing with uncertainty, Review of Economic Studies 54, 1-21. 
Hörner, J. (2004), A perpetual race to stay ahead, Review of Economic Studies 71, 1065-1088.

Judd, K.L. (2003), Closed-loop equilibrium in a multi-stage innovation race, Economic Theory 21, 673-695.

Karagözoglu, E., Sağlam, C., Turan, A. (2018), Tullock brings perseverance and suspense to tug of war, Bilkent University, March 2018, downloaded December 18, 2018, from www.researchgate.net.

Klumpp, T., Polborn, M.K. (2006), Primaries and the New Hampshire effect, Journal of Public Economics 90, 1073-1114.

Konrad, K.A. (2009), Strategy and Dynamics in Contests, Oxford University Press.

Konrad, K.A., Kovenock, D. (2005), Equilibrium and efficiency in the tug-of-war, CEPR Discussion Paper 5205.

—, - (2009), Multi-battle contests, Games and Economic Behavior 66, 256-274.

Lee, T., Wilde, L. (1980), Market structure and innovation: A reformulation, Quarterly Journal of Economics 94, 429-436.

Mago, S.D., Sheremeta, R.M. (2017), Multi-battle contests: An experimental study, Southern Economic Journal 84, 407-425.

Malueg, D.A., Yates, A.J. (2005), Equilibria and comparative statics in two-player contests, European Journal of Political Economy 21, 738-752.

—, - (2006), Equilibria in rent-seeking contests with homogeneous success functions, Economic Theory 27, 719-727.

Maskin, E., Tirole, J. (2001), Markov perfect equilibrium, Journal of Economic Theory 100, 191-219. McAfee, R.P. (2000), Continuing wars of attrition, mimeo.

McBride, M., Skaperdas, S. (2006), Explaining conflict in low-income countries; incomplete contracting in the shadow of the future, unpublished manuscript, University of California at Irvine.

Moldovanu, B., Sela, A. (2001), The optimal allocation of prizes in contests, American Economic Review 91, $542-558$

Moore, E.F. (1956), Gedanken-experiments on sequential machines, Automata Studies, Annals of Mathematical Studies 34, 129-153, Princeton University Press, Princeton NJ. 
Nti, K.O. (1999), Rent-seeking with asymmetric valuations, Public Choice 98, 415-430.

Organski, A.F.K., Lust-Okar, E. (1997), The tug of war over the status of Jerusalem: Leaders, strategies and outcomes, International Interactions 23, 333-350.

Pemantle, R., Wilf, H.S. (2009), Counting nondecreasing integer sequences that lie below a barrier, The Electronic Journal of Combinatorics 16, \#R60, 1-7.

Reeve, H.K., Emlen, S.T., Keller, L. (1998), Reproductive sharing in animal societies: Reproductive incentives or incomplete control by dominant breeders? Behavioral Ecology 9, 267-278.

Roberts, M., Samuelson, L. (1988), An empirical analysis of dynamic, nonprice competition in an oligopolistic industry, RAND Journal of Economics 19, 200-220.

Rubinstein, A. (1986), Finite automata play the repeated prisoner's dilemma, Journal of Economic Theory 39, 83-96.

Salant, Y. (2011), Procedural analysis of choice rules with applications to bounded rationality, American Economic Review 101, 724-748.

Szymanski, S. (2003), The economic design of sporting contests, Journal of Economic Literature 41, $1137-1187$.

Taylor, C.R. (1985), Digging for golden carrots: An analysis of research tournaments, American Economic Review 85, 872-890.

Tullock, G. (1980), Efficient rent-seeking, pp. 97-112 in: Buchanan, J., Tollison, R., Tullock, G., Toward a Theory of the Rent-Seeking Society, College Station: Texas A\&M Press.

Wright, B., Rodenberg, R.M., Sackmann, J. (2013), Incentives in best of $N$ contests: Quasi-Simpson's paradox in tennis, International Journal of Performance Analysis in Sport 13, 790-802.

Zizzo, D.J. (2002), Racing with uncertainty: A patent race experiment, International Journal of Industrial Organization 20, 877-902. 